\title{
Foxp3 drives oxidative phosphorylation and protection from lipotoxicity
}

\author{
Duncan Howie, ${ }^{1}$ Stephen Paul Cobbold, ${ }^{1}$ Elizabeth Adams, ${ }^{1}$ Annemieke Ten Bokum, ${ }^{1}$ \\ Andra Stefania Necula, ${ }^{1}$ Wei Zhang, ${ }^{2}$ Honglei Huang, ${ }^{3}$ David J. Roberts, ${ }^{3,4}$ Benjamin Thomas, ${ }^{1}$ \\ Svenja S. Hester, ${ }^{1}$ David J. Vaux, ${ }^{1}$ Alexander G. Betz, ${ }^{1}$ and Herman Waldmann ${ }^{1}$ \\ ${ }^{1}$ Sir William Dunn School of Pathology, ${ }^{2}$ Nuffield Department of Obstetrics and Gynaecology, John Radcliffe Hospital, \\ ${ }^{3}$ Radcliffe Department of Medicine, John Radcliffe Hospital, ${ }^{4}$ National Health Service Blood and Transplant, John Radcliffe \\ Hospital, University of Oxford, Oxford, England, United Kingdom.
}

Tregs can adopt a catabolic metabolic program with increased capacity for fatty acid oxidationfueled oxidative phosphorylation (OXPHOS). It is unclear why this form of metabolism is favored in Tregs and, more specifically, whether this program represents an adaptation to the environment and developmental cues or is "hardwired" by Foxp3. Here we show, using metabolic analysis and an unbiased mass spectroscopy-based proteomics approach, that Foxp3 is both necessary and sufficient to program Treg-increased respiratory capacity and Tregs' increased ability to utilize fatty acids to fuel oxidative phosphorylation. Foxp3 drives upregulation of components of all the electron transport complexes, increasing their activity and ATP generation by oxidative phosphorylation. Increased fatty acid $\beta$-oxidation also results in selective protection of Foxp $3^{+}$cells from fatty acidinduced cell death. This observation may provide novel targets for modulating Treg function or selection therapeutically.

Conflict of interest: The authors declare that no conflict of interest exists.

Submitted: June 17, 2016 Accepted: December 30, 2016 Published: February 9, 2017

Reference information: JCI Insight. 2017;2(3):e89160. doi:10.1172/jci.insight.89160.

\section{Introduction}

A central requirement of a functional immune system is that tolerance of host tissues is maintained, while sterilizing immunity to pathogenic microorganisms is achieved to pathogenic microorganisms. Tregs expressing the transcription factor Foxp3 play a fundamental role in maintenance of tolerance (1-3). Tregs develop in the thymus (nTregs) (1-3) and in the periphery (pTregs) $(4,5)$. Additionally, Tregs can be generated by ectopically expressing Foxp3 constructs in a T cell line (cTreg) $(3,6,7)$. They can also be induced in vitro by activation in the presence of TGF $\beta$ (iTreg) $(8,9)$. Foxp3 expression can be induced in peripheral naive $\mathrm{T}$ cells by TGF $\beta$ in multiple contexts, such as in low glucose and high lipid concentrations (10), conditions of subimmunogenic antigen presentation (11), or decreased essential amino acid concentration, which permits Treg induction partially via mTOR inhibition $(10,12)$.

Tregs have altered metabolism compared with conventional T cells. Foxp3 $3^{+}$Tregs isolated directly from mice show an increase in fatty acid-fueled oxidative phosphorylation (OXPHOS) (13) and increased spare respiratory capacity (SRC) when compared with naive CD4 T cells (14). SRC is the extra respiratory capacity available to the cell to be used upon increased energy demand.

Because Foxp3 induction occurs in many different cellular contexts, it is unclear to what extent the Treg metabolic phenotype is directed by Foxp3 alone rather than other elements of the microenvironment wherein they are generated. Two major unaddressed questions remain. Firstly, whether increased SRC and fatty acid metabolism are simply adaptations in response either to direct environmental triggers such as TGF $\beta$ or to indirect cues driven by inhibition of mTOR, or whether Treg, in otherwise replete nutrient conditions, are somehow "hardwired" to use lipid-fueled OXPHOS. Second, what is the physiological purpose for increased fatty acid oxidation by Tregs? Answering these questions may help an understanding of the relationship between the immunological function and metabolic capacity of the Treg subsets.

Several groups, including ours, have reported global transcriptome comparisons of Foxp $3^{+}$and Foxp3 CD4 ${ }^{+} \mathrm{T}$ cells $(6,15-18)$. ChIP-chip analysis suggests that Foxp3 binds to the promoters of approximately 1,200 genes (17), the majority being suppressed by Foxp3 in activated T cells (Tact). Such studies have proven limited in revealing the cell biological changes that Foxp3 exerts at the transcriptional level. To date, there have been no reported attempts to characterize the proteome controlled via Foxp3, as opposed to a 
more general Treg/Teff comparison. This is mostly due to technical limitations of mass spectrometry, as well as a lack of suitable cellular systems to dissect Foxp3 effects from those of $\mathrm{T}$ cell activation and the influential cytokine TGF $\beta$. To address this, we designed an unbiased mass spectrometry/gene set enrichment-based approach to identify common groups of proteins modulated by Foxp3 expression in all Treg types analyzed, irrespective of activation or TGF $\beta$ exposure.

We show, for the first time to our knowledge, that nuclear Foxp3 function is sufficient to program upregulation of multiple electron transport components. This increases SRC and OXPHOS activity for multiple substrates, including lipids in T cells. It does this in conditions replete for glucose $(20 \mathrm{mM})$ and amino acids, and independently of exogenous TGF $\beta$ or mTOR inhibition, although the latter is an additive signal for OXPHOS. Subsequent increased fatty acid metabolism results in reduced sensitivity to fatty acid-induced apoptosis, which is reversible by inhibition of fatty acid catabolic enzymes. These data imply that Tregs are programmed by Foxp3 to have flexibility in fuel choice, in addition to gaining a survival advantage in environments with elevated fatty acids.

\section{Results}

Foxp3 programs alterations in metabolic flux in Tregs. We first asked whether Foxp3 was sufficient to alter the capacity for OXPHOS and glycolysis in Tregs $(10,13,14,19,20)$. We compared iTregs with Tact and Foxp3 $^{+}\left(\right.$TGF $\beta$ Foxp3 $^{+}$) CD4 ${ }^{+}$T cells from iTreg cultures with Foxp3- (TGF $\beta$ Foxp3 ${ }^{-}$) cells from the same culture. The Foxp3 $3^{-}$cells are those $\mathrm{CD}^{+}{ }^{+} \mathrm{T}$ cells that did not become Foxp3 ${ }^{+}$in the presence of DCs, cognate peptide, and TGF $\beta$, and thus represent a good control for metabolism changes controlled by TGF $\beta$ alone in the absence of Foxp3 expression. For these experiments, we used $\mathrm{T}$ cells from the Foxp3 reporter mouse Marilyn.RAG ${ }^{-/}$Foxp3hCD2/CD52knockin (henceforth called MARKI). Using the Seahorse bioanalyzer, we observed that Tact and Foxp $3^{+}$iTregs had an elevated basal respiration rate when compared with Foxp3 $3^{-} \mathrm{T}$ cells from iTreg cultures, as demonstrated by a $25 \%$ increased oxygen consumption rate (OCR), an indicator of increased OXPHOS (Figure 1A). Upon addition of oligomycin, to inhibit the ATP synthase, OCR decreased to the same extent in all 3 cell types, indicating that OXPHOS was fully coupled to ATP synthesis in these cells. Uncoupling of the electron transport system (ETS) from OXPHOS using the protonophore Carbonyl cyanide-4-(trifluoromethoxy) phenylhydrazone (FCCP) reveals the maximal respiratory capacity of the cell.

Foxp $3^{+}$iTregs had approximately twice the maximal respiration of Foxp3- Tact, and 70\% more respiratory capacity than the TGF $\beta$-experienced cells that were Foxp3 $3^{-}$(Figure 1A). In addition, TGF $\beta$ experienced cells - Foxp $3^{+}$more than Foxp $3^{-}$- had higher SRC (i.e., uncoupled FCCP-induced capacity minus basal capacity) than Tact. These data suggest, perhaps, that TGF $\beta$-induced Foxp3 may be necessary for the observed increase in SRC in iTregs. To test whether the presence of nuclear Foxp3 alone was sufficient to drive a significant increase in mitochondrial respiration, we used cFoxp3 expressing EL4 T cells. EL4 T cells expressing a tamoxifen (4'hydroxy-tamoxifen, 4'HT) conditional eGFP-Foxp3-ERT (cFoxp3; EL4T. cFoxp3)) construct were cultured with or without 4'HT. In this stable cell line, administration of 4'HT results in rapid nuclear localization of cFoxp3, without exogenous TGF $\beta$ or mTOR inhibition (Figure 1B). Addition of 4'HT to EL4 cells not expressing the conditional Foxp3 construct had no discernible effect on metabolism (unpublished results). EL4.cFoxp3 cells had equivalent expression of Foxp3 to natural Tregs (Figure 1B, right panel). Nuclear Foxp3 alone, driven by tamoxifen, was sufficient to induce a significant increase in mitochondrial respiration, with increased ATP production in cFoxp3-expressing EL4 T cells exposed to 4'HT but not in control EL4 cells. However, in EL4 cells, 4'HT did not drive the increase in SRC (Figure 1C). The augmented OXPHOS induced by Foxp3 required continued Foxp3 expression in the nucleus. EL4.cFox3 cells cultured for 5 days in the presence of 4'HT had increased OXPHOS. Removal of 4'HT at day 5 led to a reduction in OXPHOS to untreated levels at day 7 (Figure 1D). No significant difference in glycolysis, as measured by extracellular acidification rate, was seen in cFoxp3-expressing EL4 cells or the parental EL4 cell line following 4'HT treatment compared with untreated cells (Figure 1, E and F). Foxp3 comprises 4 separate subdomains with different functions, the proline rich domain (ProR), the zinc finger $(\mathrm{ZnF})$, the coiled coil (CC) domain, and the forkhead (FKH) domain (Figure 1G). Although much emphasis has been placed on the FKH domain for function due to many IPEX mutations lying in that region, recent evidence shows that the ProR, primarily responsible for cofactor recruitment, is required for much of Foxp3-mediated gene regulation (21). We expressed Foxp3 with a complete deletion of the FKH domain (Foxp3 $\triangle \mathrm{FKH}$ ) or ProR (Foxp3 $\triangle$ ProR) in EL4 T cells. This revealed a requirement for both regions 

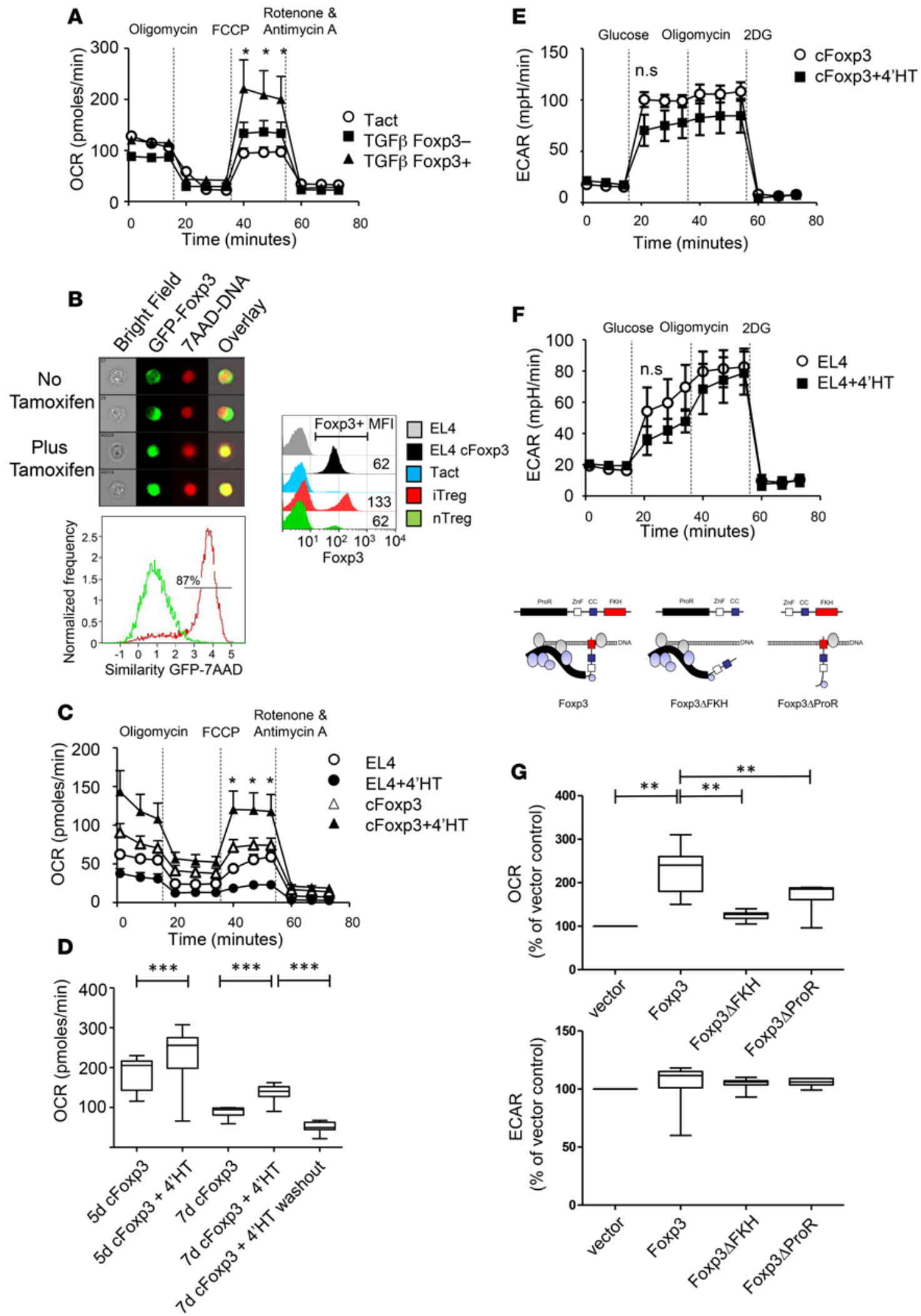

Figure 1. Foxp3 drives an increase in mitochondrial respiration. (A) Oxygen consumption rate (OCR), a measure of OXPHOS, in flow cytometry-sorted Marilyn.foxp3hCD2 knockin Foxp3 ${ }^{+}$iTreg compared with Foxp3- cells or Marilyn.foxp3hCD2 knockin T cells activated (Tact) in the absence of TCF $\beta$. Results representative of 3 separate experiments. Means \pm SD. ${ }^{*} P<0.05$ by 2 -way ANOVA. (B) Left panel; EL4.cFoxp3 cells were cultured overnight with or without $50 \mathrm{nM}$ 4'HT. Measurement of nuclear versus cytoplasmic eGFP by Imagestream. The percentage similarity for 7AAD and eGFP (Foxp3) was measured for EL4.cFoxp3 without (green histogram) and with (red histogram) 4'HT treatment. Right panel: Foxp3 expression in EL4, EL4.cFoxp3, Tact, CD4 ${ }^{+} \mathrm{T}$ cells from iTreg induction cultures and nTreg. (C) OCR of cFoxp3-expressing EL4 cells with and without 4'HT for 48 hours, compared with 
EL4 cells without the cFoxp3 construct. Results representative of 3 separate experiments. Means \pm SD. ${ }^{*} P<0.05$ by 2 -way ANOVA. (D) OCR of EL4. cFoxp3 cells cultured for 5 days in the presence or absence of 4'HT (first 2 bars). The cells were washed at day 5 and cultured for a further 2 days with or without (washout) 4'HT. 7d cFoxp3 indicates cells cultured for 7 days without 4'HT; 7d cFoxp3 + 4'HT indicates cells cultured for 7 days with 4'HT. Results representative of 2 separate experiments. Boxes span 25th to 75 th percentiles, whiskers represent minimum and maximum values, and horizontal line shows median. ${ }^{* *} P<0.0001$ by 2 -way ANOVA. (E and $\mathbf{F}$ ) Extracellular acidification rate (ECAR) in cFoxp3-expressing EL4 cells and EL4 cells without cFoxp3 with or without 4'HT. ECAR in response to glucose, oligomycin, and 2'deoxyglucose (2DG) after 48 hours with or without 4'HT. Results representative of 3 separate experiments. Means \pm SD. (C) Top panel: EL4 cells transfected with retroviral constructs encoding Foxp3, Foxp3 lacking the forkhead domain "Foxp3 $\Delta$ FKH", or Foxp3 lacking the proline-rich region "Foxp3 $\Delta$ ProR". Empty vector was used as a negative control. ProR, proline-rich region; ZnF, zinc finger; CC, coiled coil region; FKH, Forkhead region. Middle panel: OCR of EL4 cells transfected with the indicated constructs. Data represent percentage change relative to empty vector, pooled results of 2 separate experiments. ${ }^{*} P<0.005$, Student's $t$ test. Bottom panel: ECAR of EL4 cells transfected with the indicated constructs, pooled results of 2 separate experiments. Boxes span 25th to 75 th percentiles, whiskers represent minimum and maximum values, and horizontal line shows median.

in intact Foxp3 to impart the augmented respiration (Figure 1G, middle panel), but the measurements had no effect on glycolysis (Figure 1G, bottom panel). This result suggests that, in order for Foxp3 to program increased OXPHOS, it requires both direct DNA binding and recruitment of ProR binding partners. Taken together, these experiments show that Foxp3 expression alone drives increased ETS capacity, leading to an increase in both basal respiration and in SRC.

Foxp3-mediated OXPHOS and mTOR activation. mTOR, a serine threonine kinase, is the major nutrient sensor of the cell and, upon activation, can induce cells to upregulate glycolysis and anabolic metabolism (22). We asked whether Foxp3-controlled mitochondrial respiration might require mTOR in cFoxp3-expressing EL4 cells. First, we titrated the concentration of rapamycin in EL4 and EL4.cFoxp3 cells to find the optimal amount to inhibit mTOR activation. We found that $5 \mathrm{nM}$ decreased ribosomal $\mathrm{S} 6$ protein phosphorylation to an equivalent extent in both cell lines (Supplemental Figure 1A; supplemental material available online with this article; https://doi.org/10.1172/jci.insight.89160DS1). An equivalent sensitivity to rapamycin is seen in natural Tregs and Tconv (Supplemental Figure 1C).

Inhibition of mTOR, confirmed by decreased phospho-S6 (Supplemental Figure 1B), resulted in a small increase in basal respiration in cFoxp3-expressing cells (Figure 2A), but 4'HT and rapamycin treatment combined to significantly increase both the basal respiration and the SRC in cFoxp3-expressing cells (Figure 2A) in an additive fashion. Thus, mTOR inhibition and Foxp3 expression in EL4 cells can work in concert to increase OXPHOS, but mTOR inhibition is not essential for Foxp3 to enhance OXPHOS. Next, we investigated the role of mTOR activation in Foxp3-mediated OXPHOS regulation in TGF $\beta$-treated primary T cells from MARKI mice. Cells activated for 7 days with cognate antigen in the presence or absence (Tact) of TGF $\beta$ were flow sorted based on surface human CD2 prior to 24 hours of rapamycin treatment. In primary $\mathrm{T}$ cells, basal rates of OXPHOS were inhibited by mTOR inhibition irrespective of TGF $\beta$ exposure (Figure 2, B-D), although basal and maximal rates of OXPHOS were higher in Foxp3-expressing cells. The degree of SRC was significantly different between Tact and TGF $\beta$-exposed cells. Rapamycin inhibited SRC in TGFß-exposed cells but moderately enhanced SRC in Tact. These data suggest that the mechanism of augmented OXPHOS induced by Foxp3 expression is independent of the status of mTOR activation.

OXPHOS fueled by fatty acid $\beta$-oxidation is enhanced by Foxp 3 expression. The mitochondrial ETS can accept electrons from various substrates at multiple sites on the 5 constituent complexes (Figure 3A). We tested whether Foxp3 expression was responsible for augmenting the activity of all ETS members or whether some complexes were more responsive to their substrates than others in Foxp3-expressing cells. EL4.cFoxp3 cells treated or not with 4'HT, and suspended in buffer with no respiration substrates, were treated with digitonin to selectively permeabilize the plasma membrane, thus allowing respiration substrates to leak out of the cell. Cells were then supplied with substrates for electron transport complex I, fatty acids or pyruvate; II succinate; and IV, N,N,N',N'-Tetramethyl-p-Phenylenediamine (TMPD) in ascorbate (Figure 3A). OXPHOS increased in response to addition of each of these ETC substrates to a greater degree in EL4.cFoxp3 cells following 48 hours of 4'HT treatment (Figure 3B). This increase is probably not due to changes in mitochondrial number, as 4'HT was not found to change the mitochondrial/genomic DNA ratio (Supplemental Figure 2A). Thus, Foxp3 expression is alone sufficient to increase the cell's ability to drive OXPHOS in response to multiple substrates, including fatty acids. Tregs have been shown to be preferentially induced in conditions where glucose metabolism is inhibited and fatty acids are abundant (10). EL4 cells (Figure 3C) and EL4.cFoxp3 cells (Figure 3D) were treated with 4'HT 

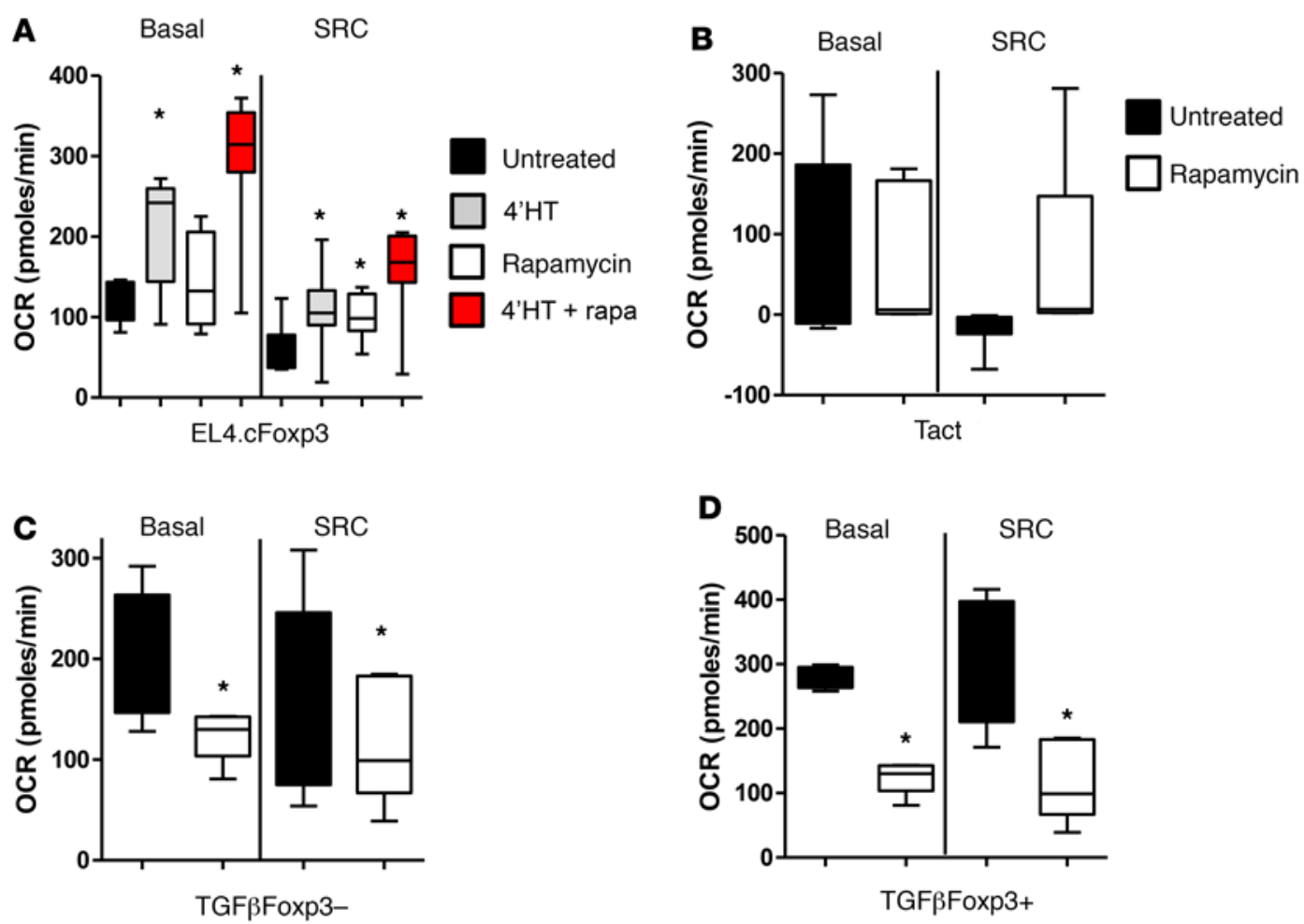

Figure 2. mTOR inhibition and Foxp3 expression act independently to regulate OXPHOS. (A) Basal and spare respiratory capacity (SRC) of EL4.cFoxp3 T cells following 4'HT, rapamycin, or combination 4'HT+rapamycin treatment for 48 hours. Results representative of 3 experiments. In all panels, boxes span 25th to 75th percentiles, whiskers represent minimum and maximum values, and horizontal line shows median. ${ }^{*} P<0.05$ by Student's $t$ test. (B) Basal and SRC of activated Marilyn.foxp3hCD2 knockin CD4+ T cells (Tact). Cells were activated for 7 days with DCs and peptide before flow sorting and overnight treatment with $5 \mathrm{nM}$ rapamycin. Results representative of 2 experiments. (C and D) Basal and SRC of activated Marilyn.foxp3hCD2 knockin CD4 ${ }^{+} \mathrm{Foxp3}^{+}$and Foxp3- T cells. Cells were activated for 7 days with DCs and peptide in the presence of TCF $\beta$ before flow sorting for CD4 and CD2 and overnight treatment with $5 \mathrm{nM}$ rapamycin. Results representative of 2 experiments. ${ }^{*} P<0.05$ by Student's $t$ test.

for 48 hours prior to treatment with combinations of BSA-conjugated palmitate (a long-chain fatty acid) and etomoxir (an inhibitor of the transporter carnitine palmitoyl transferase 1A [cpt1a]), which inhibits uptake of palmitate into the mitochondrion. Addition of etomoxir inhibited OXPHOS in EL4.cFoxp3 cells but not in EL4 cells in the absence of exogenous fatty acids (Figure 3D). This implies that nuclear Foxp3 expression enables these cells to metabolize endogenous fatty acids. Addition of BSA-palmitate significantly increased maximal respiration ("0" versus "palm" black bars) in EL4.cFoxp3 cells in an etomoxir-reversible fashion but did not do so in the parental cell line. This shows that Foxp3 drives a preferential increase in the metabolism of fatty acids in these cells. Addition of palmitate or etomoxir had a negligible effect on glycolysis in EL4 or EL4.cFoxp3 cells (data not shown). Thus, Foxp3 augments OXPHOS fueled by fatty acids in cells that had been grown in the presence of sufficient glucose and amino acids.

Foxp3 expression induces increased transcription of multiple mitochondrial protein-encoding genes. To investigate whether Foxp3 expression results in upregulation of components of OXPHOS at the mRNA level, we used quantitative PCR (qPCR) arrays. We isolated mRNA of flow sorted Foxp3 ${ }^{+}$and Foxp3 ${ }^{-}$cells from iTreg induction cultures. Foxp3- were those cells exposed to TGF $\beta$ that did not convert to Foxp $3^{+}$, around $30 \%-50 \%$ of the cells in an iTreg in vitro induction culture. We compared Foxp3-mediated changes in these cells with those observed in cFoxp3-expressing EL4 cells treated or not with 4'HT. In this way, we aimed to discriminate between those transcripts controlled by Foxp3 from those modulated by TGF $\beta$ independently of Foxp3. mRNA was analyzed using commercial qPCR arrays based on mitochondrial and glycolysis pathway-associated protein transcripts. Foxp3 was found to drive a significant upregulation of the 84 mitochondrial-associated gene transcripts at the population level (Figure 4, A-C). There was, however, a wide variation in transcript numbers between the genes for different ETS subcomponents, and many genes were slightly downregulated (Figure 4C). Additionally, the concordance between EL4 and primary T cells was 
A

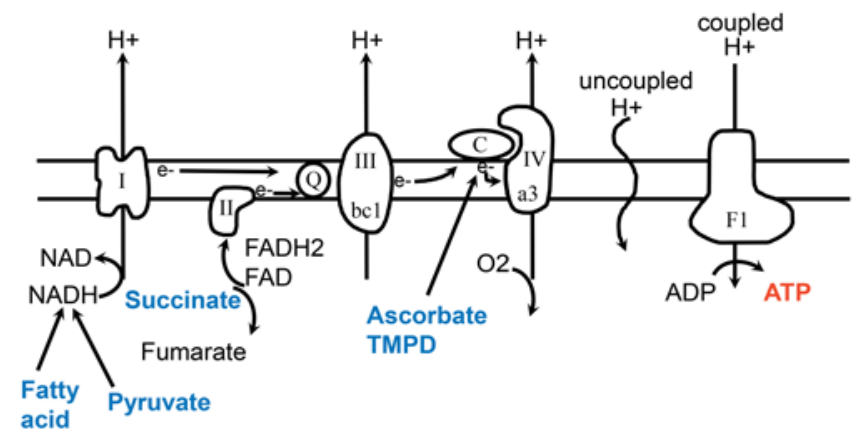

B

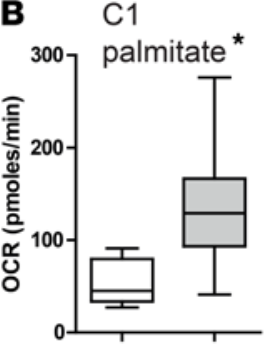

C1

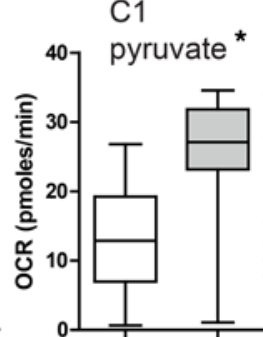

$\mathrm{C} 2$

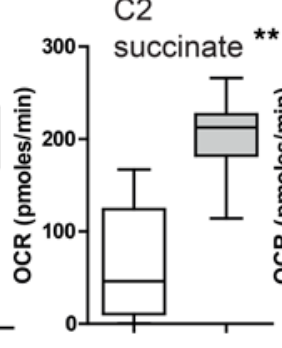

$\mathrm{C} 4$

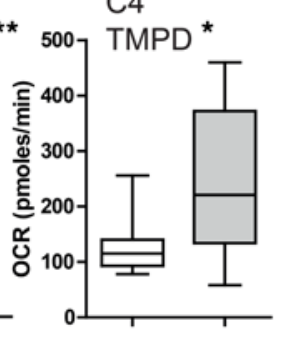

Figure 3. Respiratory substrate usage by cFoxp3-expressing EL4 T cells. (A) The electron transport system with the exogenous experimental substrates added and their contribution to the electron transport highlighted in blue. (B) Increase in oxygen consumption rate induced by palmitate, pyruvate, succinate, and TMPD in digitonin-permeabilized EL4 (white bars) and EL4.cFoxp3 (gray bars) cells. Experiment representative of 3 similar experiments. Means \pm SEM. ${ }^{*} P<0.05,{ }^{* *} P<0.01$ by Student's $t$ test. (C and D) Basal (white bars), maximal (black bars), and basal/maximal (red bars) oxygen consumption rate of EL4 (c) and EL4cFoxp3 T cells cultured with 4'HT with the addition of BSA-conjugated palmitate (palm), etomoxir (eto), or BSA-palmitate plus etomoxir. Results representative of 3 separate experiments. Boxes span 25th to 75th percentiles, whiskers represent minimum and maximum values, and horizontal line shows median. ${ }^{*} P<0.05$, ${ }^{* *} P<$ $0.01,{ }^{* *} P<0.001$ by Student's $t$ test.
C

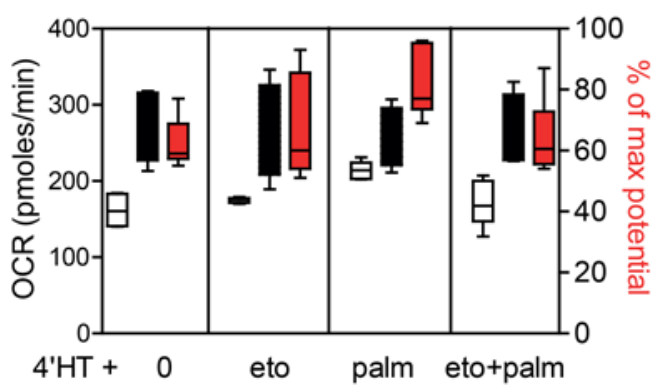

D

EL4 cFoxp3

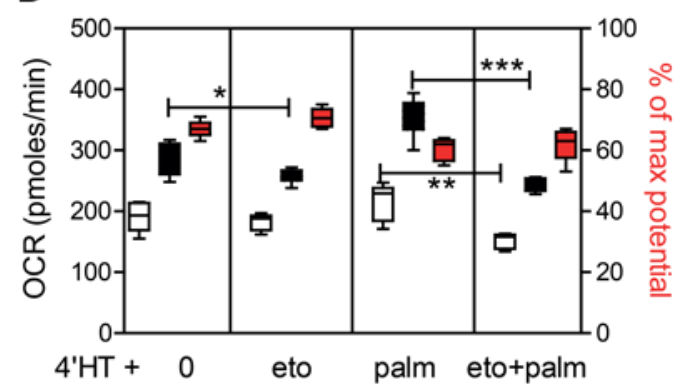

$\square$ Basal OCR
Max OCR
Basal/max OCR

quite weak, with many transcripts being regulated in opposite ways between the two cell types. Nuclear Foxp $^{+}$cells from EL4.cFoxp3 cultures displayed significantly higher expression of components of all 5 complexes of the ETS: ndufab1 (complex I), sdha (complex II), uqcrc1 (complex III), cox4i2, cox6b2, cox7a2, cox $8 c$ (complex IV), and atp $4 b$ (complex V) compared with non-4'HT-treated cells, implying that at least some of these complexes may be under direct transcriptional control. We performed a new gene set enrichment analysis on microarray data (previously published in Regateiro et al.; ref. 6). Comparison of MARKI Foxp $3^{+}$and Foxp3- iTregs with Foxp3 $3^{-/} \mathrm{CD}^{+} \mathrm{T}$ cells cultured in identical conditions showed that only the Foxp $^{+}$iTregs were enriched in transcripts associated with Treg signatures, including FOXP3, NRP1, and SELL, as expected (Supplemental Figure 3). There was also a significant association with genes involved in metabolism of lipids (Gene Ontology classification, "cellular lipid metabolic processes"), but gene sets associated with glycolysis and oxidative phosphorylation did not reach statistical significance. These data point to a general role for Foxp3 in enhancing transcription of OXPHOS genes in the absence of additional polarizing signals, but clear differences exist between EL4 and primary T cells.

The proteome of cells expressing Foxp3 is enriched for mitochondrial ETS proteins. The global correlation between mRNA transcript number and protein abundance is generally weak in most cells (reviewed in ref. 23). Because of this, and because of the differences in Foxp3-influenced ETS mRNA seen between EL4 and primary T cells, we investigated Foxp3-influenced changes at the protein level. We quantified the global proteomic landscape of mouse Tregs governed by Foxp3 expression with 3 comparative quantitative mass spectrometry experiments (Figure 5A and Supplemental Figure 4A). First, to compare iTregs with Tact, 
A

\section{iTreg}
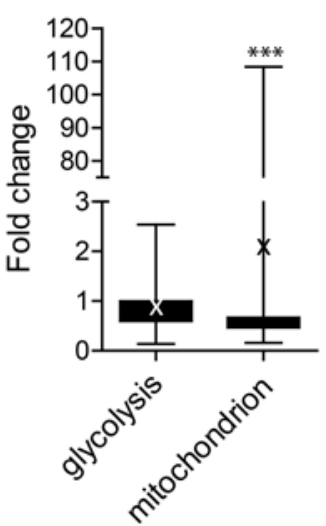

B

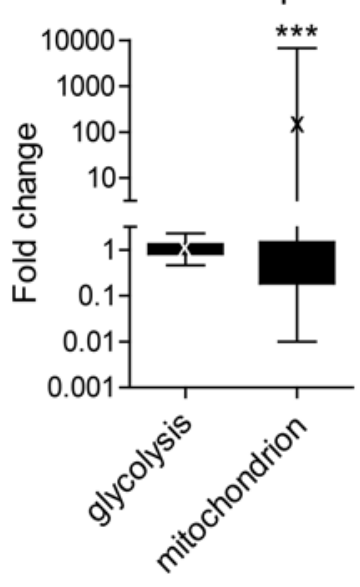

$=1.0$

Glycolysis transcripts

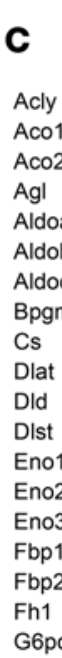

C

$\begin{array}{cl}\text { iTreg EL4 } & \\ \text { Foxp3+ } & \text { cFoxp3 }\end{array}$

/Foxp3- +/- tam

Aco2

Aldob

Aldoc

Bpgm

Dlat

Did

Dist

Eno2

Eno3

Fbp1

Fbp2

G6pc

G6pc3

G6pdx

Galm

Gapdhs

Gbe1

Gck

Gpi1

Gsk3a

Gsk3b

Gys1

Gys2

H6pd

Hk2

$\mathrm{Hk} 3$

Idh1

Idh2

Idh3a

Idh3b

Idh3g

Mdh1

Mdh1b

Mdh2

Ogdh

\section{iTreg EL4}

Foxp3+ cFoxp3

/Foxp3- +/- tam

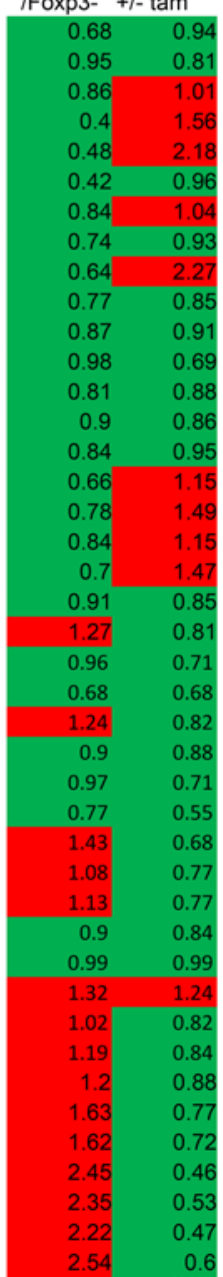

Mitochondrial transcripts

\begin{tabular}{|c|c|c|c|c|c|}
\hline & $\begin{array}{l}\text { iTreg } \\
\text { Foxp3+ } \\
\text { /Foxp3- }\end{array}$ & $\begin{array}{l}\text { EL4 } \\
\text { cFoxp3 } \\
+/- \text { tam }\end{array}$ & & $\begin{array}{l}\text { iTreg } \\
\text { Foxp3+ } \\
\text { /Foxp3- }\end{array}$ & $\begin{array}{l}\text { EL4 } \\
\text { cFoxp3 } \\
+/- \text { tam }\end{array}$ \\
\hline Atp12a & 0.61 & 1.54 & Ndufa3 & 0.46 & 0.75 \\
\hline Atp4a & 0.57 & 0.12 & Ndufa4 & 0.65 & 0.19 \\
\hline Atp $4 b$ & 0.16 & 2083.32 & Ndufa5 & 0.49 & 0.14 \\
\hline Atp5a1 & 0.55 & 0.35 & Ndufa6 & 0.68 & 0.17 \\
\hline Atp5b & 1.02 & 0.09 & Ndufa7 & 0.91 & 0.32 \\
\hline Atp $5 \mathrm{c} 1$ & 0.53 & 0.1 & Ndufa8 & 0.56 & 0 \\
\hline Atp5d & 13.9 & 0.6 & Ndufab1 & 0.67 & 10.42 \\
\hline Atp5f1 & 108.38 & 0.2 & Ndufb10 & 0.52 & 0.43 \\
\hline Atp5g1 & 0.61 & 0.65 & Ndufb2 & 0.64 & 0.43 \\
\hline Atp5g2 & 0.47 & 0.19 & Ndufb3 & 0.56 & 0.11 \\
\hline Atp5g3 & 0.57 & 0.24 & Ndufb4 & 0.55 & 0.81 \\
\hline Atp5h & 0.33 & 0.2 & Ndufb5 & 0.52 & 0.19 \\
\hline Atp5j & 0.47 & 0.12 & Ndufb6 & 0.54 & 0.79 \\
\hline Atp5j2 & 0.38 & 0.19 & Ndufb7 & 0.74 & 0.46 \\
\hline Atp5o & 0.43 & 0.01 & Ndufb8 & 0.58 & 0.25 \\
\hline Atp6v0a2 & 0.43 & 0.01 & Ndufb9 & 0.6 & 0.12 \\
\hline Atp6v0d2 & 0.49 & 0.75 & Ndufc1 & 0.53 & 0.96 \\
\hline Atp6r1c2 & 0.45 & 0 & Ndufc2 & 0.52 & 0.05 \\
\hline Atp6v1e2 & 0.23 & 0.48 & Ndufs1 & 0.61 & 0.67 \\
\hline Atp6v1g3 & 0.35 & 87.7 & Ndufs2 & 0.71 & 0.2 \\
\hline Bcs1l & 0.47 & 0.05 & Ndufs3 & 0.68 & 0.29 \\
\hline Cox11 & 0.4 & 65.25 & Ndufs 4 & 0.62 & 0.97 \\
\hline Cox4i1 & 0.98 & 0 & Ndufs5 & 0.87 & 3.49 \\
\hline Cox4i2 & 0.42 & 6675.53 & Ndufs6 & 0.53 & 8.58 \\
\hline Cox5a & 0.42 & 0 & Ndufs7 & 0.66 & 0.18 \\
\hline Cox5b & 0.49 & 209.09 & Ndufs8 & 0.68 & 0.51 \\
\hline Cox6a1 & 0.5 & 0.07 & Ndufv1 & 0.66 & 0.55 \\
\hline Cox6a2 & 0.82 & 0.29 & Ndufv2 & 0.67 & 0.57 \\
\hline Cox6b1 & 0.46 & 0 & Ndufv3 & 0.53 & 0.1 \\
\hline Cox6b2 & 0.47 & 2333.05 & Oxa1l & 0.79 & 0.13 \\
\hline Cox6c & 0.42 & 0 & Ppa1 & 0.85 & 0.27 \\
\hline Cox7a2 & 0.5 & 783.98 & Ppa2 & 0.53 & 1.8 \\
\hline Cox7a2I & 0.43 & 0.4 & Sdha & 0.65 & 11.78 \\
\hline Cox7b & 0.48 & 0.23 & Sdhb & 0.54 & 0.43 \\
\hline Cox8a & 1.16 & 0 & Sdhc & 0.38 & 0.03 \\
\hline Cox8c & 1.39 & 2141.89 & Sdhd & 0.59 & 2.39 \\
\hline Cyc1 & 0.47 & 0.02 & Uqcr11 & 0.74 & 2.24 \\
\hline Lhpp & 0.61 & 1.61 & Uqcre1 & 0.55 & 12.68 \\
\hline Ndufa1 & 0.52 & 0.21 & Uqcrc2 & 0.53 & 0.26 \\
\hline Ndufa10 & 0.44 & 0.43 & Uqcrfs1 & 1.27 & 0.07 \\
\hline Ndufa11 & 0.56 & 0.31 & Uqcrh & 0.5 & 6.41 \\
\hline Ndufa2 & 0.64 & 0.54 & Uqcrq & 0.56 & \\
\hline
\end{tabular}

Figure 4. Foxp3 exerts transcriptional control on multiple mitochondrial genes. Comparison of Foxp3 positive and negative $T$ cells polarized under iTreg conditions and EL4 cells expressing conditional Foxp3 treated with and without tamoxifen by qPCR array. Data for A-C represent the mean of qPCR performed separately on 3 biological replicates, then pooled. (A) Comparison of the fold change of all the glycolytic gene set transcripts with the mitochondrial component gene set transcripts in Foxp3 positive and negative iTreg by 2-way ANOVA. Boxes span 25th to 75th percentiles, whiskers represent minimum and maximum values, and horizontal line shows median. " $X$ " marks mean, ${ }^{* * *} P<0.005$. (B) Comparison of the fold change of all the glycolytic gene set transcripts with the mitochondrial component gene set transcripts in nuclear Foxp3 ${ }^{+}$versus cytoplasmic Foxp3 ${ }^{+}$EL4 T cells by 2-way ANOVA. Boxes span 25th to 75 th percentiles, whiskers represent minimum and maximum values, and horizontal line shows median. " $X$ " marks mean, ${ }^{* * *} P<0.005$. (C) Heat map of qPCR array data. Glycolysis- and mitochondrial-associated genes, as well as their fold changes, are listed. EL4 fold change indicates the ratio of 4'HT to non-4'HT-treated cells. iTreg fold change indicates the ratio of CD2 ${ }^{+} \mathrm{RAG}^{-1-M a r i l y n}$.Foxp3hCD2 to CD2-cells. Red indicates transcripts with a ratio $>1.00$, and green indicates transcripts with a ratio $<1.00$. tam, tamoxifen.

Foxp $3^{+} \mathrm{CD} 4^{+} \mathrm{T}$ cells from iTreg cultures using $\mathrm{T}$ cells from MARKI mice were compared with activated cells in the absence of TGF $\beta$. Using GSEA comparison of iTregs with Tact MARKI T cells revealed in iTregs an upregulation of OXPHOS-associated proteins, with the most significantly enriched gene set being "respiratory electron transport" (Figure 5, B and C, left panel). The leading edge proteins, those contributing most to the significance of the association, included proteins of the mitochondrial ETS complex I (the NDUFs), complex II (SDHA, SDHB), complex III (CytB), and complex IV (COX2, COX5A). To distinguish between the effects of TGF $\beta$ versus Foxp3 expression on this result, the second experiment made a comparison of Foxp3 positive and negative cells from MARKI iTreg cultures in the same manner (Figure 5, B and C, middle panel). The most significant gene set association in the iTreg Foxp3 ${ }^{+}$versus Foxp3comparison was KEGG Parkinson's disease, the leading edge proteins of which comprise subunits of ETS complex I, II, III, and IV of the ETS and the mitochondrial ATP synthase (ATP5E). These data suggest that 
A

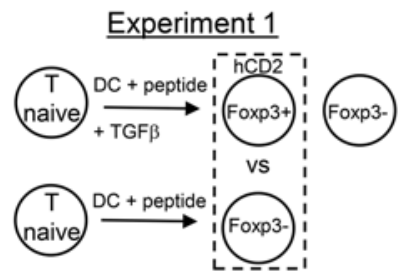

Experiment 2

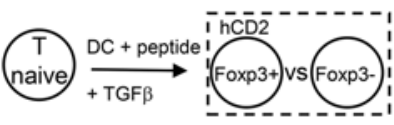

Experiment 3

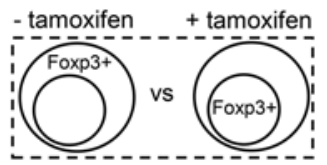

C Experiment 1

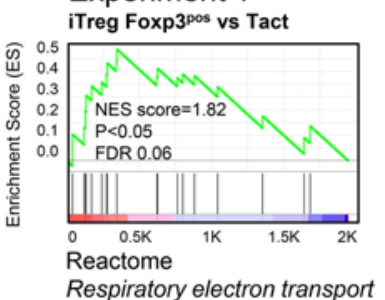

B

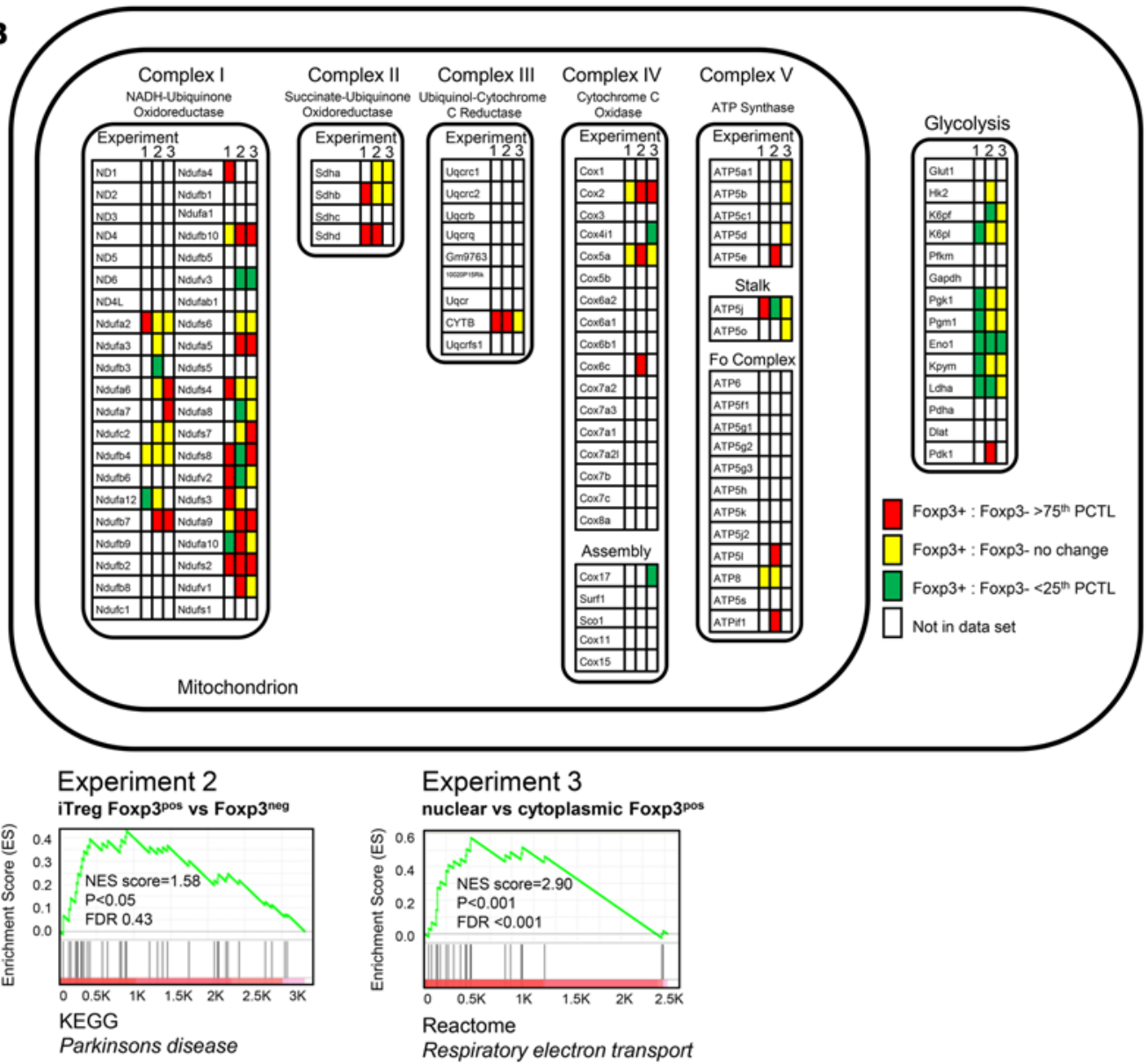

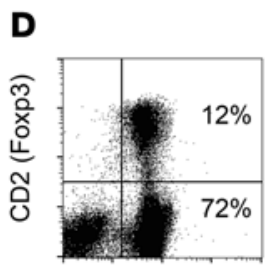

CD4

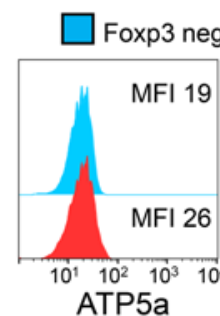

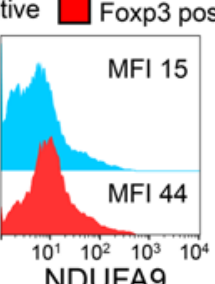

NDUFA9

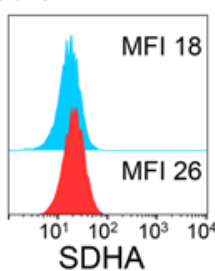

Figure 5. Foxp3 expression enhances expression of electron transport system proteins. (A) Foxp3 expression experiments for proteomic analysis. Cell comparisons used to quantitate proteomic differences between Foxp3+ and Foxp3- cells. (B) Electron transport system protein subunit comparison between Foxp3 positive and negative T cells. Red boxes indicate proteins with a Foxp3+/Foxp3- ratio in the 75 th percentile of all proteins measured. Green boxes indicate proteins with a Foxp3 $3^{+} /$Foxp3 $^{-}$ratio in the 25 th percentile of all measured proteins. Yellow boxes indicate proteins with no change between Foxp3+ or Foxp3-. White boxes indicate proteins not present in the proteome. Figure adapted from http://wikipathways.org/index.php/ Pathway:WP295. (C) Gene set enrichment analysis of the mass spectroscopy data from experiments 1-3. NES, normalized enrichment score. $P$ values calculated using a weighted Kolmogorov-Smirnov test. (D) Flow cytometric measurement of ATP5a, NDUFA9, and SDHA on permeabilized splenic $\mathrm{CD}^{+}{ }^{+} \mathrm{CD2}{ }^{-}$and $\mathrm{CD} 4{ }^{+} \mathrm{CD2}{ }^{+}$cells from C57BL/6.foxp3hCD2 knockin mice. Data representative of 2 separate experiments.

TGF $\beta$ might signal increased OXPHOS only in cells where Foxp3 is induced. To test the role of Foxp3 in the absence of other Treg features, cFoxp3 expressing EL4 T cells treated or untreated with 4'OH tamoxifen were compared. Using this reductionist approach, the most significantly overrepresented group of proteins in EL4 cells with nuclear Foxp3 included components of ETS I, II, and III (Figure 5, B and C, right panel). EL4 cells not expressing the Foxp3 construct did not change expression of these complexes with the addition of 4'HT (unpublished data). Thus, Foxp3 drives upregulation of OXPHOS proteins in the presence of (saturating) $20 \mathrm{mM}$ glucose in the absence of exogenous TGF $\beta$. We used flow cytometry to measure expression of NDUFA9, SDHA, and ATP5a as representative components of ETS complexes I, II, and V, respectively, in nTregs and Tconv (Figure 5D). The 3 proteins were more highly expressed in Tregs than in Foxp3- cells. 


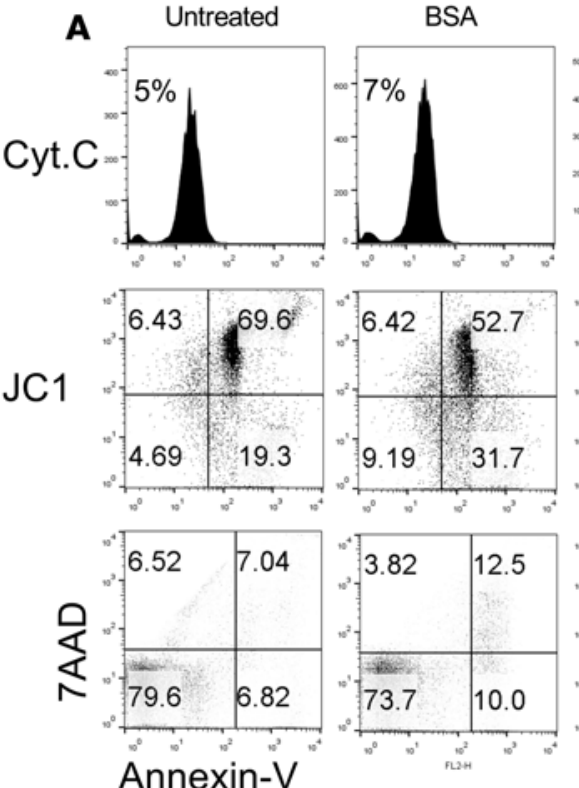

Annexin-V

B

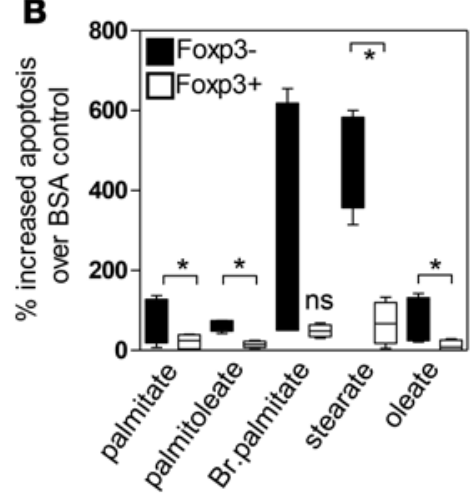

C

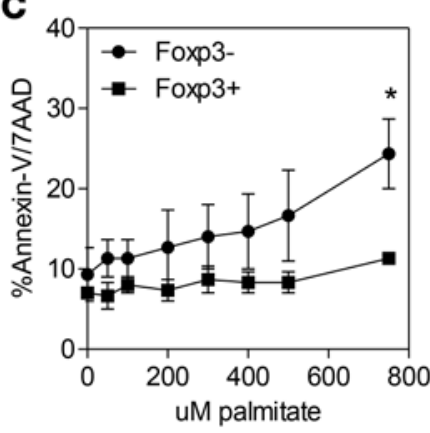

Br.palmitate (16:0) palmitoleate (16:1) stearate (18:0)
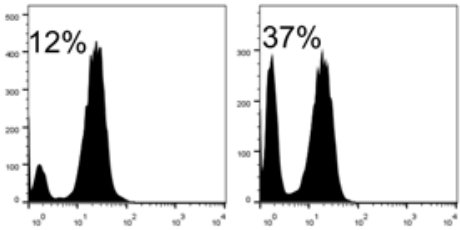

oleate $(18: 1)$
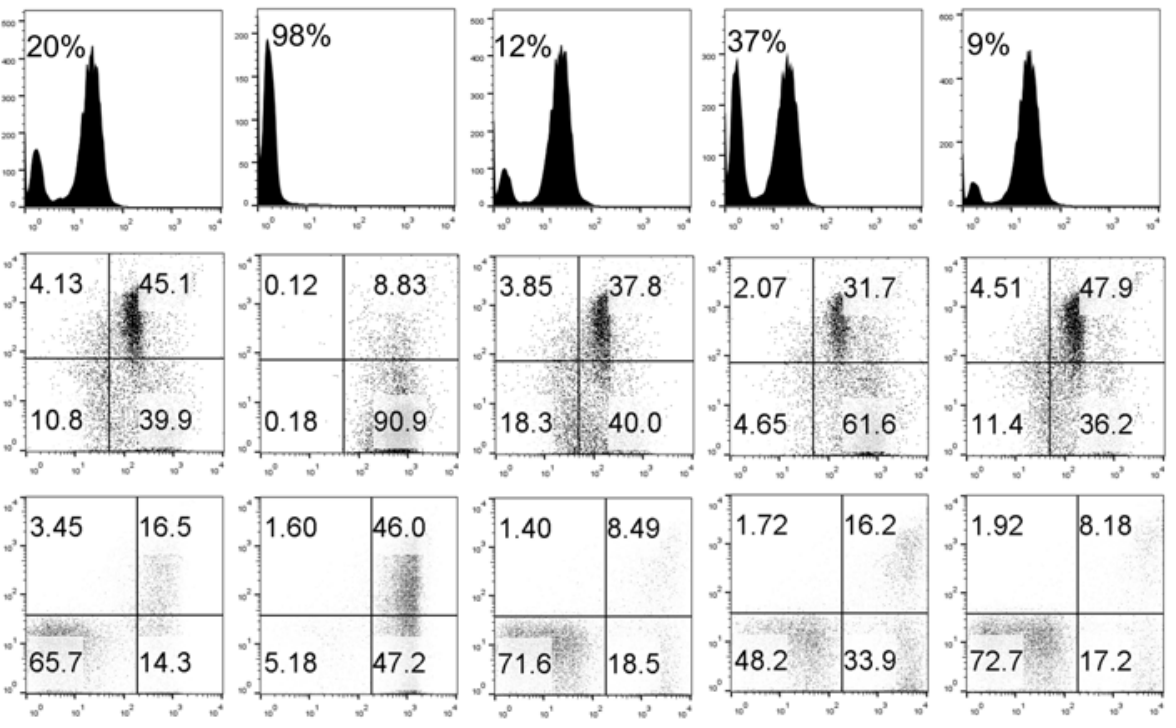

D
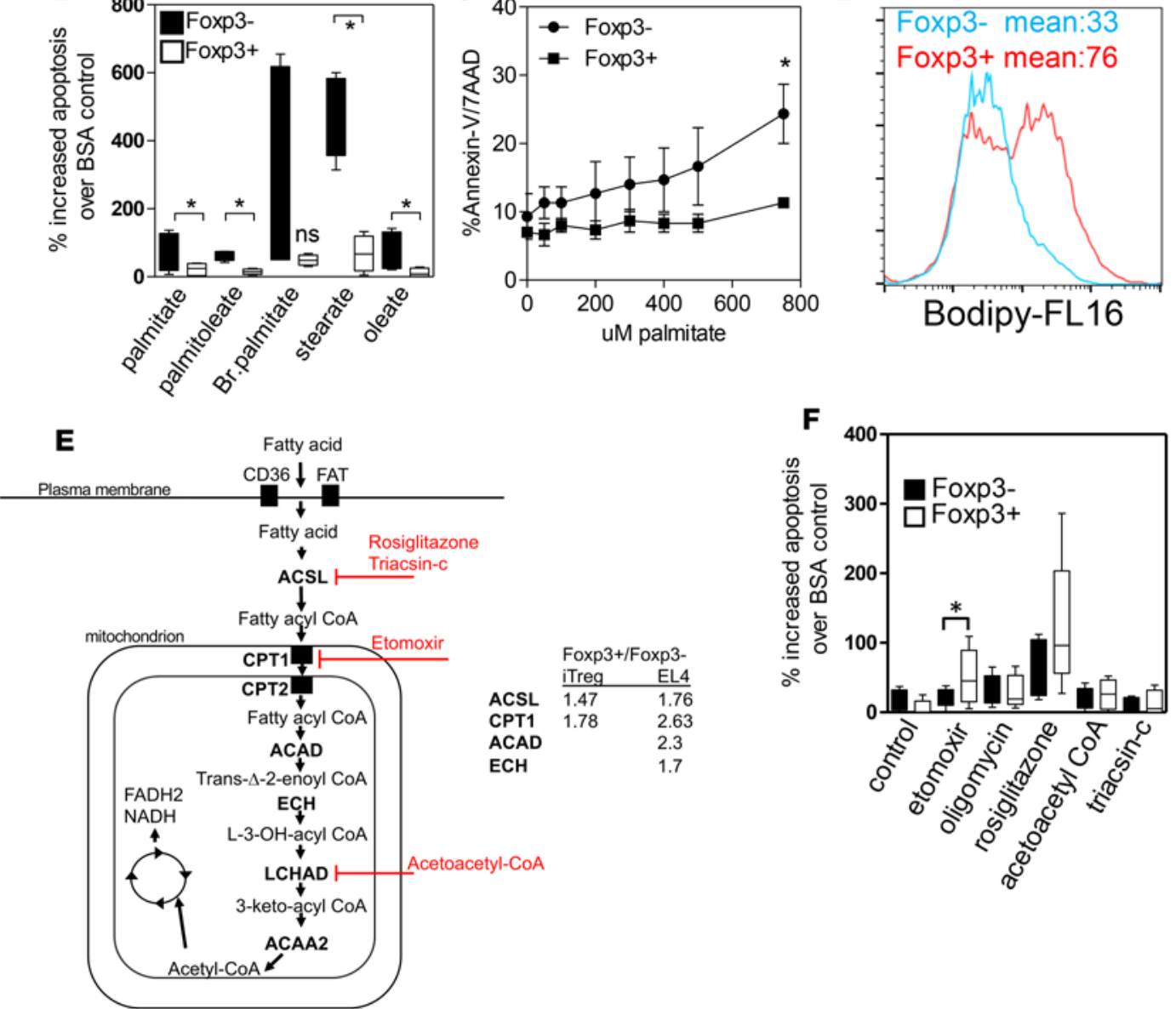

Figure 6. Foxp3-driven fatty acid $\beta$-oxidation protects Tregs from long-chain fatty acid-mediated apoptosis. (A) C57BL/6 CD4 ${ }^{+}$T cells were cultured for 18 hours in the presence of IL-2 $(5 \mathrm{U} / \mathrm{ml})$ and IL-7 $(10 \mathrm{ng} / \mathrm{ml})$, in addition to the indicated BSA-conjugated long-chain fatty acid at a concentration of $500 \mu \mathrm{M}$. Apoptosis was measured by mitochondrial cytochrome c release (Cyt.C), mitochondrial loss of mitochondrial membrane potential ( $\Delta \psi \mathrm{m}$, JC1 stain), and 7AAD/annexin V staining according to Methods. Results representative of 2-5 separate experiments. (B) C57BL/6. Foxp3hCD2/CD52 knockin $\mathrm{CD}^{+} \mathrm{T}$ cells were cultured for 18 hours with IL-2, IL-7, and the indicated BSA-conjugated long-chain fatty acid before flow cytometric analysis of surface human CD2 (Foxp3) and 7AAD/annexin V positivity. Results representative of 4 pooled experiments. Boxes span 25th to 75 th percentiles, whiskers represent minimum and maximum values, and horizontal line shows median. ${ }^{*} P<0.05$ by Student's $t$ test. (C) Titration of BSA-conjugated palmitate. Cells cultured as in B with titrated amounts of BSA-conjugated palmitate prior to flow analysis of surface human CD2 and 7AAD/annexin V positivity. 
Results pooled from 3 biological repeats. Means \pm SEM. ${ }^{*} P<0.05$ by Student's $t$ test. (D) Uptake of BODIPY-labeled palmitate by CD4 ${ }^{+} T$ cells from C57BL/6.Foxp3hCD2/CD52 knockin mice. Results representative of 3 separate experiments. (E) Schematic of fatty acid $\beta$-oxidation pathway showing major enzymes and their inhibitors used in this study. Inset: ratiometric data (Foxp3+/Foxp3-) from mass spectrometry analysis of the major $\beta$-oxidation enzymes, where present, in MARKI iTreg and EL4cFoxp3 T cells. (F) C57BL/6. Foxp3hCD2/CD52 knockin CD4+ $T$ cells were cultured for 18 hours with IL-2, IL-7, and BSA-conjugated palmitate with the addition of the indicated inhibitors before flow cytometric analysis of surface human CD2 (Foxp3) and $7 A A D / a n n e x i n V$ positivity. Results pooled from 3 separate biological repeats. Boxes span 25 th to 75 th percentiles, whiskers represent minimum and maximum values, and horizontal line shows median, ${ }^{*} P<0.05$ by Student's $t$ test.

We next asked whether Foxp3 ${ }^{+}$human $\mathrm{CD} 4^{+} \mathrm{T}$ cells were similarly enriched for proteins involved in mitochondrial respiration. Human peripheral blood $\mathrm{CD} 4^{+} \mathrm{T}$ cells were stimulated for 24 and 48 hours with anti-CD3 in order to separate the CD25 and Foxp3 $3^{+}$and Foxp3 ${ }^{-}$cells into distinct populations for efficient flow cytometric sorting (Supplemental Figure 4, A and B). Prior to TCR stimulation, we found CD4 ${ }^{+} \mathrm{T}$ cells in human PBMCs consisted of CD25-Foxp3-, CD25 ${ }^{+}$Foxp3 $^{-}$, and $1.5-8 \%$ of CD25 $5^{+}$Foxp3 ${ }^{+}$subpopulations. After 24-hour TCR stimulation, these 3 subpopulations became further segregated, as expression of CD25 and Foxp3 were upregulated. Particularly, both CD25 and Foxp3 were upregulated in a discrete population, which consisted of $9 \%-21 \%$ of $C D 4^{+} \mathrm{T}$ cells. Both percentage and maximal intensity of CD25 expression was further upregulated after 48-hour TCR stimulation, indicating that T cell activation became generalized. There was also a doubling of the percentage of CD25 Foxp3 ${ }^{+}$cells. Unlike CD25, the maximal intensity of Foxp3, however, remained unchanged. Since human T cells require around 72 hours of TCR stimulation to start cell division, this further increase in percentage of CD25+Foxp $3^{+}$cells at 48 hours is unlikely to derive from Treg proliferation but, instead, from Foxp3 induction in conventional T cells. For this reason, we analyzed cells at 24 after activation.

Cells sorted on the basis of the topmost $10 \%$ of CD4, CD25, and FOXP3 in 24-hour stimulated cells were found to be quite different with respect to significantly different gene sets. A three-way comparison revealed that only the triple-positive cells $\left(\mathrm{Foxp}^{+}\right)$upregulated mitochondrial proteins (Supplemental Figure 4C). We conclude, therefore, that the upregulation of OXPHOS proteins is a property of Foxp3 in both mouse and man.

Increased ETS protein production in response to Foxp3 expression did not appear to be due to an increase in mitochondrial biomass. EL4 cells or EL4.cFoxp3 cells cultured with 4'HT did not increase their mitochondrial DNA/genomic DNA ratio, and MARKI iTregs cultured with increasing doses of TGF $\beta$ did not demonstrate increased mitotracker staining measured by mean fluorescence intensity (Supplemental Figure 2). Thus, the increased ETS protein induced by Foxp3 is likely on a per-mitochondrion basis. These proteomic data provide a molecular basis for the observed increased OXPHOS capacity of Foxp $3^{+}$cells.

Enhanced lipid metabolism protects Tregs from fatty acid-induced cell death. The physiological benefit derived from Foxp3-driven increased long-chain fatty acid metabolism in Tregs is unclear. It is possible that it imparts a survival advantage to memory Tregs in low glucose/glutamine conditions due to increased flexibility of fuel choices. Another possibility is that augmented $\beta$-oxidation of long-chain fatty acids protects the cells from their well described proapoptotic effects (24). To test this hypothesis, we first conjugated saturated and unsaturated $\mathrm{C} 16$ (palmitate $\mathrm{C} 16: 0$, palmitoleate $\mathrm{C} 16: 1$, and nonmetabolizable bromo palmitate $\mathrm{C} 16: 0$ ) and $\mathrm{C} 18$ (stearate $\mathrm{C} 18: 0$ and oleate $\mathrm{C} 18: 1$ ) fatty acids to BSA to facilitate their uptake into cells and tested their toxicity on $\mathrm{CD}^{+} \mathrm{T}$ cells. Resting $\mathrm{T}$ cells cultured with BSA-conjugated fatty acids were induced to enter apoptosis to varying degrees as measured by mitochondrial cytochrome c release; loss of mitochondrial membrane potential $(\Delta \psi \mathrm{m})$ measured by JC-1 staining where red/green fluorescence indicates high/low $\Delta \psi \mathrm{m}$, respectively; and annexin $\mathrm{V} / 7 \mathrm{AAD}$ staining (Figure $6 \mathrm{~A}$ ). Bromo palmitate, which is unable to undergo $\beta$-oxidation, induced a high level of mitochondrial cytochrome $\mathrm{c}$ release, along with a markedly decreased mitochondrial membrane potential. Palmitate, palmitoleate, stearate, and oleate all induced apoptosis, with stearate inducing the highest level of cytochrome c release, decreased $\Delta \psi \mathrm{m}$, and decreased annexin V positivity. We next asked whether Foxp3 ${ }^{+}$and Foxp3 ${ }^{-}$CD $4^{+} \mathrm{T}$ cells differed in their susceptibility to palmitate-induced apoptosis. CD4 ${ }^{+} \mathrm{T}$ cells from C57B1/6.Foxp3hCD2/CD52 knockin mice containing both $\mathrm{Foxp}^{+}$and $\mathrm{FoxP}^{-}$cells were cultured in the presence of BSA-conjugated fatty acids for 18 hours, and Foxp3 and annexin V/7AAD positivity were measured. All fatty acids tested induced a significant increase in apoptosis in Foxp3- cells at 18 hours, with palmitate and stearate inducing 2- to 5 -fold increases in apoptosis, respectively, compared with BSA alone (Figure 6B). There was significantly less fatty acid-induced apoptosis observed in Foxp $3^{+}$cells, with palmitate- and stearate-treated Foxp $3^{+}$cells 
exhibiting 2- to 4-fold less apoptosis. As expected, Br-palmitate, which is both resistant to $\beta$-oxidation and inhibits mitochondrial fatty acid metabolism, induced equal apoptosis of Foxp3 positive and negative cells. Palmitate-induced apoptosis showed a linear dose response within the physiological range starting at 50 $\mu \mathrm{M}$ and increasing to the highest concentration tested $(800 \mu \mathrm{M})$. Within this range, Foxp $3^{+}$cells were uniformly resistant to apoptosis (Figure 6C), commensurate with their increased fatty acid oxidation. Analysis of BODIPY-labeled palmitate uptake by Tregs revealed that they have the capacity to take up substantially more palmitate than conventional $\mathrm{T}$ cells, as indicated by a shift in intensity of BODIPY staining (Figure 6D). Finally, we asked whether Foxp3 might program cells to resist fatty acid-induced apoptosis by upregulating components of the fatty acid metabolism pathway, illustrated in Figure 6E. Our mass spectroscopy data showed that conditional Foxp3 expression in EL4 cells induced increased acyl-CoA synthetase-long (ACSL), carnitine palmitoyl transferase-1 (CPT1), Acyl-CoA dehydrogenase (ACAD), and Enoyl-CoA hydratase (ECH). TGF $\beta$-induced iTregs expressing Foxp3 had enhanced expression of ACSL and CPT1 compared with Foxp3 ${ }^{-}$cells (Figure 6E). We tested whether inhibition of the fatty acid $\beta$-oxidation pathway or of electron transport with specific inhibitors would reverse the Treg resistance to palmitate-induced apoptosis. Inhibition of ACSL with triacsin-c or rosiglitazone, CPT1 with etomoxir, or LCHAD with acetoacetyl-CoA reversed the resistance of Tregs to palmitate-induced apoptosis, as did inhibition of the mitochondrial ATPase with oligomycin (Figure 6F).

Collectively, these data demonstrate the mechanism by which Foxp3 exerts a metabolic flexibility on $\mathrm{T}$ cells, imparting increased ability to utilize fatty acids as fuel for OXPHOS, and at the same time endows them with increased resistance to cell death induced by long-chain fatty acids.

\section{Discussion}

In the present study, we showed that Foxp3 is capable of altering the proteome of the cell in such a way as to increase the total ETS capacity in addition to lipid oxidation capacity in the absence of exogenous TGF $\beta$. Nuclear localization of Foxp3 alone was sufficient to increase the cells' maximal respiration using palmitate as the only extracellular substrate. As a consequence, increased fatty acid oxidation may confer a survival advantage to Tregs in the presence of proapoptotic concentrations of long-chain fatty acids.

$\mathrm{T}$ cells adjust their metabolism in response to availability of nutrients in the form of glucose, glutamine, and fatty acids. The balance of extracellular metabolism substrates can also have significant effects on the development of $\mathrm{T}$ cell subsets $(10,19,20)$. As both memory and Tregs preferentially adopt lipid-fueled OXPHOS, it has not been clear until now whether the adaptation of Treg metabolism was due to general mTOR inhibition or a consequence of Foxp3 reprogramming. The latter case would imply that cell intrinsic metabolic control in Tregs is important functionally.

We found that Foxp3 expression has a powerful effect on enhancing the mitochondrial respiration of the cell. This occurs both in ectopically expressing Foxp3 and in TGF $\beta$-induced Foxp $3^{+}$primary T cells. Foxp3 expression in either context results in upregulation of many ETS proteins of complexes I-V. Most of the upregulated proteins are common to both cell types. The regulation of the ETS mRNA transcripts shares much less similarity between EL4 cells and primary T cells. Regulation of ETS subunit gene transcription is a complex process involving a cascade of transcription factors. Peroxisome proliferator activated receptor $\gamma$ coactivator a (PGC-1 $\alpha)$ is known to be required for their transcription and itself induces the transcription of 15-20 different transcription factors (25) - including ERR $\alpha$, SIRT3, and GABPA - which have been shown to act as transcription factors for several ETS genes (26). SIRT3 and PGC-1 $\alpha$ are critical for Treg function in the setting of experimental heart transplantation (27). It is likely that the differences in ETS transcripts between the different cell types and differences between protein and mRNA levels arise from a combination of epigenetic differences, differences in regulation of PGC-1a downstream transcription factors, different Foxp3 binding partners, and posttranscriptional regulation.

In order to become activated and to proliferate, T cells must undergo a major increase in energy expenditure for increased biomass production. The increased energy requirement of Tact is provided mostly through glycolysis (28-30). Glycolysis is an amphibolic process that allows rapid $\mathrm{T}$ cell expansion by providing NADPH and ribose for reductive biosynthetic reactions and synthesis of nucleotides, and it fuels lipid synthesis via citrate from the TCA cycle. T cells primarily use glucose and glutamine to fuel this rapid expansion but can switch to use of ketone bodies and fatty acids under resting conditions (31).

Tregs in vitro appear as relatively quiescent cells and, as such, might be expected to have a lower requirement for amphibolic metabolism. However, in vivo Tregs are capable of rapid expansion while maintaining 
suppressive function $(32,33)$. Tregs have been shown to express less cell-surface Glut1 than Tconv, due to Foxp3-mediated inhibition of Akt (34), and for functional activity, Treg expression of Glut1 seems to be redundant (35). We have found that Foxp3 programs an increased capacity for OXPHOS and fatty acid oxidation without any inhibition of glycolytic capacity. In this way, Tregs are able to fuel robust proliferation via anabolic metabolism at the same time as using catabolic metabolism to generate further ATP.

Human Tregs appear metabolically flexible, switching between glycolysis-only and both glycolysis and fatty acid oxidation upon activation (36). In addition to providing energy for cell survival and division, the choice between utilizing glycolytic and oxidative metabolism defines the lineage decision between Th17 cells and Tregs, respectively (13) (reviewed in ref. 37). The decision to oxidize fatty acids for OXPHOS is taken by Tregs in addition to memory T cells (14) and is influenced by nutrient status, via mTOR and AMPK activation as well as pyruvate dehydrogenase kinase 1 (PDHK1) activation, Acetyl-CoA carboxylase 1 (ACC1) expression $(13,14)$, and PTEN activity $(38,39)$. Increased uptake of fatty acids by Tregs may be explained by our observation of increased protein levels of acetyl coA synthetase (ACSL) and carnitine palmitate transferase 1A (CPT1A) influenced by Foxp3. Our data suggest that, for Foxp3 to drive increased OXPHOS, both direct DNA binding via the FKH domain and possibly indirect DNA binding via recruited cofactors at the ProR are required. TGF $\beta$ and inhibition of mTOR are inductive signals for Foxp3 expression but are dispensable for this function of Foxp3.

In addition to generating energy, there are several additional potential consequences to upregulation of the ETS in T cells. Increased reactive oxygen species production as a by-product of increased electron transport has been reported to have a negative effect on Th17 development (14) and, as such, may play an indirect positive role in Treg development. We find Tregs and Tact release ATP (data not shown), which can rapidly be converted to antiinflammatory adenosine via CD39 and CD73 (40-45). It remains to be demonstrated whether secreted ATP, rather than ATP released from dying cells, represents a significant substrate for membrane-bound ectonucleotidases. A further consequence that we have demonstrated in this study may be intracellular removal of potentially harmful oxidative substrates, such as long chain free fatty acids. We show that Foxp3-expressing cells are relatively resistant to fatty acid-induced death due to removal by $\beta$-oxidation. The combination of increased lipid uptake and increased ETS protein production with concomitant elevated fatty acid $\beta$-oxidation is cytoprotective to Tregs. This mechanism may be physiologically important in multiple ways. In human and mouse plasma, the free fatty acid concentration ranges from 200--600 $\mu \mathrm{M}$ but can be 3-fold higher in diabetes (46). Palmitate is the most abundant plasma-free fatty acid at around $25 \%(47,48)$; however, elevated plasma free fatty acid levels are associated with obesity and the metabolic syndrome and cause inflammation, activation of macrophages, and death of multiple cell types including pancreatic $\beta$ cells, neurons, and $\mathrm{T}$ lymphocytes $(24,49,50)$. It will be important to ascertain whether resistance to lipotoxicity by Tregs plays a role in survival in vivo. Treg numbers in visceral fat may increase or decline depending on multiple factors, which might include inflammatory mediators, adipose cell types, and different polarized macrophages in the adipose tissue. Human Tregs have been shown to be either increased in visceral adipose tissue (51-53), diminished (51), or increased in subcutaneous fat (53, 54). They have also been shown to be decreased in visceral fat in humans and mice $(54,55)$. Dysregulation of lipid metabolism can lead to tissue-specific inflammatory pathology. Nonalcoholic fatty liver disease has been shown to result in release of linoleic acid (C18:2) from hepatocytes, leading to preferential CD4 ${ }^{+} \mathrm{T}$ cell apoptosis via increased ROS production and decreased $\Delta \psi \mathrm{m}$ (56). Saturated fat has also been shown to potently activate innate immunity in mice with macrophages lacking a regulator of lipoprotein lipase, Angiopoietin-like protein 4 (Angpt14). In these animals, saturated fatty acids are excessively taken up by peritoneal macrophages, resulting in activation and a fatal acute phase response. The mechanism of fatty acid-induced apoptosis may involve induction of endoplasmic reticulum stress, leading to calcium leakage from the ER and decreased mitochondrial membrane polarity, release of mitochondrial cytochrome-c, and activation of the intrinsic apoptosis pathway (57).

$\mathrm{T}$ cells are exquisitely sensitive to environmental nutrient and oxygen concentrations and respond to these by the opposing actions of mTOR, AMPK, and HIF1 $\alpha$ to adapt to their environment. Conditions of nutrient starvation induce decreases in mTOR activation, which permits Foxp3 to be transcribed. We now show that once Foxp3 is present, it reprograms T cells to adapt to energy demands by enhanced oxidative phosphorylation. The ability to modify the T cells' capacity for catabolic metabolism via increased ETS component expression and oxidative phosphorylation, along with protection from apoptosis, is a newly described role for Foxp3. 


\section{Methods}

Mouse and human cells. Mice were bred and maintained in SPF conditions at the Sir William Dunn School of Pathology. All procedures were conducted in accordance with the Home Office Animals (Scientific Procedures) Act of 1986. We previously generated and described MARKI and Marilyn.Foxp $3^{-/-}$mice (5). Human peripheral blood lymphocytes were obtained from normal blood donors at the John Radcliffe Hospital with informed consent.

Untouched splenic $\mathrm{CD}^{+} \mathrm{T}$ cells were isolated by magnetic sorting using commercial kits (Miltenyi Biotec). Anti-CD4 (clone GK1.5) and CD25 (clone 3C7) were obtained from BD Biosciences. iTregs were generated from MARKI mice by culturing red blood cell-depleted splenocytes with female C57BL/6 BM-derived DCs at a ratio of 5:1 in the presence of dby peptide $(100 \mathrm{nM}), \mathrm{IL}-2$ (Peprotech, $100 \mathrm{U} / \mathrm{ml})$, all trans retinoic acid ( $10 \mathrm{nM}$ ), and TGF $\beta$ (Peprotech, $2 \mathrm{ng} / \mathrm{ml}$ ). Peptides were synthesized by the MRC peptide synthesis unit (Imperial College School of Medicine, London, United Kingdom) and were assessed for purity by HPLC. All peptides exceeded 95\% purity. Retinoic acid was purchased form Sigma Aldrich. Cells were cultured for 7 days prior to sorting, based on staining with anti-CD4 and human CD2 (clone RPA2.10, BioLegend) for Foxp3-expressing cells. Tact were cultured in the same way with the omission of TGF 3 . EL4.cFoxp3 cells were generated by transfecting EL4 cells with a construct encoding GFP-Foxp3ERT fusion protein, previously described $(6,7)$.

$q P C R$. Taqman reverse transcriptase PCR (RT-PCR) was performed essentially as described (9). Primers for glycolytic and oxidative phosphorylation-related genes were purchased as PCR arrays from Qiagen (RT2 profiler PCR array). Biological triplicate cultures for cFoxp3-expressing EL4 cells with and without 4'OH tamoxifen addition for 48 hours were performed, and qPCR was done on each separately. Likewise, triplicate iTreg cultures were performed on MARKI CD4 ${ }^{+} \mathrm{T}$ cells prior to sorting human CD2 positive and negative cells for Foxp3 positive and negative cells. Mitochondrial-to-genomic DNA measurement by qPCR was performed according to the protocol of Guo et al. (58).

Quantitative mass spectrometry. EL4 and EL4.cFoxp3 cells were labeled in culture with SILAC. Cells were grown for 7 days in complete RPMI medium, in which arginine and lysine were replaced with either unlabeled ("light") or heavy arginine $\left({ }^{13} \mathrm{C}_{6}{ }^{15} \mathrm{~N}_{4} \mathrm{~L}\right.$-arginine $\mathrm{HCl}, \mathrm{CK}$ Isotopes Ltd.) and light or heavy lysine $\left({ }^{13} \mathrm{C}_{6}{ }^{15} \mathrm{~N}_{2}\right.$ L-lysine $\mathrm{HCl}, \mathrm{CK}$ Isotopes Ltd.). Labeled and unlabeled cells were then treated or not, respectively, with 4'HT (Sigma-Aldrich) $(20 \mathrm{ng} / \mathrm{ml})$ for 5 days prior to lysis and protein solubilization in $8 \mathrm{M}$ urea. Soluble tryptic peptides from 4'HT treated and untreated samples were measured by BCA and mixed at a 1:1 ratio prior to prefractionation into 24 fractions by in-solution isoelectric focussing on an Agilent Offgel 3100 apparatus. Separated proteins were reduced and alkylated in $2 \mathrm{M}$ urea using DTT and iodoacetamide (Sigma-Aldrich) at room temperature prior to overnight digestion with trypsin at $37^{\circ} \mathrm{C}$. Peptides were acidified to $5 \%$ formic acid and desalted on c18 column (Waters Corporation) prior to drying and resuspension in $2 \%$ acetonitrile and $0.1 \%$ formic acid. Samples were injected onto an Ultimate 3000 nano HPLC (Dionex, Thermo Fisher Scientific) system coupled to an Orbitrap mass spectrometer (Thermo Electron). Samples were resolved on a $15 \mathrm{~cm}$ by 75 micron inner diameter picotip column (New Objective), which was packed in house with reprosil-Pur C18-AQ phase. A 90-minute gradient was used to separate the peptides. The mass spectrometer was operated in data-dependent acquisition mode. Precursor scans were performed in the orbitrap at a resolving power of 60,000 , from which 5 precursor ions were selected and fragmented in the linear ion trap. Charge state +1 ions were rejected. Peak lists were generated using MS Convert ProteoWizard and searched using Mascot (Matrixscience). Data were searched against IPI-Mus musculus database (http://www.matrixscience.com/help/seq_db_setup_ipi.html). Precursor mass accuracy tolerance was set at $10 \mathrm{ppm}$ and MS/MS at $0.5 \mathrm{Da}$. The EL4 data yielded, on average, 3,827 unique proteins from 2 experiments. Quantitative ratiometric data were obtained using Thermo Scientific Proteome Discoverer software v1.4.

Mouse T cells, approximately $1 \times 10^{7}$ per sample, were pelleted and lysed in sodium 3-[(2-methyl-2 undecyl-1, 3-dioxolan-4-yl)methoxy]-1-propanesulfonate (Rapigest, Waters Corporation) prior to reduction and alkylation with DTT and iodoacetamide and overnight trypsinisation. Following acidification to 5\% formic acid, the peptides were desalted on 18 nanocolumns assembled in-house. Labelling of peptides with light or heavy formaldehyde was performed on-column according to Boersema et al. (59). The mouse datasets yielded 3,074 (iTreg Foxp3 ${ }^{-}$vs. Foxp3 $3^{+}$) and 1,963 (Tact vs. iTreg Foxp3 ${ }^{+}$) unique proteins. Comparison of protein types isolated from $8 \mathrm{M}$ urea EL4 and rapigest iTreg lysates are depicted in Supplemental Figure 5. Quantitative data were obtained using ProteomeDiscoverer software (Thermo Fisher Scientific). 
Human peripheral blood mononuclear cells from normal blood donors were separated into CD4 subsets based on CD25 and Foxp3 expression by a combination of anti-CD4 beads (Miltenyi) and flow cytometric sorting. Cells $\left(1 \times 10^{7}\right)$ were lysed in RIPA buffer containing protease and phosphatase inhibitors with the aid of sonication. Proteins ( $40 \mu \mathrm{g}$ per sample) were reduced and alkylated in $6 \mathrm{M}$ urea using DTT and iodoacetamide at room temperature prior to overnight digestion with trypsin at $37^{\circ} \mathrm{C}$. Following acidification to $5 \%$ formic acid, the peptides were desalted on c18 and eluted in $65 \%$ acetonitrile, $0.1 \%$ formic acid before drying and resuspension in $2 \%$ acetonitrile and $0.1 \%$ formic acid.

Peptides $(1 \mu \mathrm{g}$ ) were separated on UPLC (Waters UPLC, nano-Acquity, $75 \mu \mathrm{m} \times 250 \mathrm{~mm}, 1.7 \mu \mathrm{m}$ particle size) coupled with MS/MS (Thermo OrbiTrap-Velos; 60,000 resolution; top 20 precursor ion selection for collision-induced dissociation [CID]) workflow and a gradient of $1 \%-40 \%$ Acetonitrile in 120 minute at a flow rate of $250 \mathrm{nl} / \mathrm{min}$. Acquired MS/MS data were converted into an MGF file by using Thermo Convert software and then submitted to Central Proteomics Facilities Pipeline-Oxford (CPFP-Oxford) for protein identification. Protein quantitation was performed with normalized spectral index quantitation (SINQ) (60). Gene set enrichment analysis was performed as previously described (61). GSEA examines whether a defined set of genes are significantly enriched at the top or bottom of a ranked list of genes from microarray or proteomic experiments, which represent 2 phenotypes (e.g., Foxp3 ${ }^{+}$and Foxp3- cells). Preranked analysis was performed on ranked ratiometric data from labeled quantitative mass spectrometry data derived from Proteome Discoverer output. Gene sets interrogated included KEGG, Reactome, and Biocarta. The mass spectrometry proteomics data have been deposited to the ProteomeXchange Consortium (62) via the PRIDE partner repository with the dataset identifiers PXD001789 (mouse) and PXD001906 (human).

Measurement of metabolic flux. Cellular metabolism was measured using an XF96 cellular flux analyzer instrument from Seahorse Bioscience. OXPHOS was measured using a Mitostress test kit (Agilent Technologies) according to the manufacturer's instructions. Primary T cells and T cell lines $\left(3 \times 10^{5}\right.$ per well $)$ were cultured in RPMI with no sodium bicarbonate and 1\% FCS, $20 \mathrm{mM}$ glucose, $2 \mathrm{mM}$ pyruvate, and 50 $\mu \mathrm{M} \beta$ mercaptoethanol ( $\mathrm{pH} \mathrm{7.4)}$ ) at $37^{\circ} \mathrm{C}$ for these assays. Glycolysis was measured both via ECAR measurements from the mitostress test data and by using Glyco stress test kits. Metabolism of fatty acid was measured by starving the cells in low glucose $(2 \mathrm{mM})$ medium containing $0.5 \mathrm{mM}$ carnitine and $1 \%$ fatty acid-free FCS for 2 hours followed by a 1-hour starvation period in Krebs-Henseleit buffer supplemented with $2.0 \mathrm{mM}$ glucose, $0.5 \mathrm{mM}$ carnitine, and $5 \mathrm{mM}$ HEPES ( $\mathrm{pH}$ 7.4). Fifteen minutes prior to assay, the cells were treated or not with $40 \mu \mathrm{M}$ etomoxir, and immediately prior to the assay, the cells were supplied with BSA-palmitate $(150 \mathrm{mM})$ or BSA as a control.

Metabolic flux measurements with permeabilized cells. Extracellular flux analysis in permeabilized cells was performed as described in ref. 63, with some modifications. Cells were plated on poly-1-lysine-coated seahorse 96 -well plates at 300,000 cells per well, permeabilized in digitonin ( $25 \mu \mathrm{g} / \mathrm{ml}$ final) in mannitol and sucrose (MAS)-BSA buffer with $1 \mathrm{mM} \mathrm{ADP}$ for 10 minutes at $37^{\circ} \mathrm{C}$ before starting measurements. Individual electron transport substrates were injected following 3 basal measurements. Substrates used and final concentrations were palmitoyl-1-carnitine $(50 \mu \mathrm{M})$, pyruvate $(20 \mathrm{mM})$, succinate $(20 \mathrm{mM})$, TMPD, and ascorbate $(0.25 \mathrm{mM}$ and $1.04 \mathrm{mM})$. All chemicals were from Sigma-Aldrich.

Foxp3 mutant retroviral constructs. Foxp3 mutants cloned into the retroviral vector M6P are described in ref. 21. These constructs are described in ref. 21. EL4 cells were transiently transfected with electroporation using a Bio-Rad genepulser, with $10 \mu \mathrm{g}$ DNA per transfection at a voltage of $250 \mathrm{~V}$ and a capacitance of $970 \mu \mathrm{F}$. Cells were analyzed 24 hours following transfection and were routinely $50 \%$ transfected at this time.

Western blotting. Cell lysis was carried out with ice-cold lysis buffer (Tris $20 \mathrm{mM}$ [pH 7.6], $\mathrm{NaCl} 140$ mM, EDTA $2 \mathrm{mM}$, NaF $0.5 \mathrm{mM}$, sodium orthovanadate $1 \mathrm{mM}$, $\beta$ glycerophosphate $25 \mathrm{mM}$, sodium pyrophosphate $2 \mathrm{mM}$, benzamidine $2 \mathrm{mM}$, DTT $0.5 \mathrm{mM}$, and protease inhibitors (Complete, EDTA-free Protease Inhibitor Cocktail; Roche Diagnostics) containing 0.5\% Triton X-100. Cell lysates were clarified by centrifugation at $14,000 \mathrm{~g}$ for 15 minutes at $4^{\circ} \mathrm{C}$. Lysates were subjected to SDS-PAGE, using approximately 10 $\mu \mathrm{g}$ protein per lane and transferred onto PVDF filters (Invitrogen). Filters were blocked for 1 hour with $5 \%$ skim milk or $5 \%$ BSA and then probed with the indicated antibodies. Bound antibody was revealed using horseradish peroxidase-conjugated secondary antibodies using enhanced chemiluminescence (Supersignal, Pierce Biotechnology). Anti-phospho-S6 ribosomal protein (Ser235/236, clone 2F9, catalog 4858) and anti-S6 ribosomal protein (clone 5G10, catalog 2217) were purchased from Cell Signalling Technology.

Preparation of albumin-bound-free fatty acids. Fatty acids $(0.2 \mathrm{M})$ in ethanol were diluted 1:25 into Krebs Ringer bicarbonate HEPES buffer ( $\mathrm{pH} 7.4$ ) containing $20 \%$ BSA at $60^{\circ} \mathrm{C}$ with gentle agitation for 30 minutes 
prior to dilution in complete culture medium to the desired working concentration. Molar ratio of fatty acid/BSA was kept at less than 3 to maintain fatty acid/BSA binding.

Measurement of apoptosis. Annexin V/7AAD staining was performed using kits from BioLegend (PE Annexin V Apoptosis Detection Kit with 7-AAD) and eBioscience (Annexin V Apoptosis Detection Kit) according to the manufacturers' directions. Mitochondrial cytochrome c release was measured according to the method of Campos et al. (64) using $0.0005 \%$ digitonin to selectively permeabilize the $\mathrm{T}$ cell plasma membrane. Mouse monoclonal anti-cytochrome c $(6 \mathrm{H} 2 . \mathrm{B} 4$, Novus Biologicals) was detected with a secondary anti-mouse immunogloubulin conjugated to Alexa Fluor 647 (BioLegend). Mitochondrial membrane potential $(\Delta \psi \mathrm{m})$ was measured with JC-1 dye (Thermo Fisher Scientific) by flow cytometry according to the manufacturer's directions.

Statistics. All statistics were performed as indicated using GraphPad Prism 6 for Windows, version 6.05. Student's $t$ test was used to compare 2 groups; in analysis where multiple groups were compared, ANOVA was performed. Significance was defined as $P<0.05$.

Study approval. All animal procedures were conducted in accordance with the Home Office Animals (Scientific Procedures) Act of 1986 under project licence number 30/3060, and the local ethical review panel, the central Committee on Animal Care and Ethical Review (ACER), at the University of Oxford. Human Tregs were isolated either from leucocyte cones or leukapheresis obtained from the National Blood and Transplant Services under institutional ethics approval. Additional Ethics permission was obtained from the South Central Oxford Research Ethics Committee (08/H0607/41) for leukapheresis collections to be made from normal donors. All participants signed the written consent form to participate in this study.

\section{Author contributions}

DH, SPC, AGB, and HW designed research studies; DH, EA, ATB, ASN, WZ, HH, DJR, BT, and SSH conducted experiments; DH, ATB, ASN, WZ, HH, DJR, BT, and SSH acquired data; DH, WZ, HH, DJR, $\mathrm{BT}, \mathrm{DJV}$, and $\mathrm{HW}$ analyzed data; AGB provided reagents; and $\mathrm{DH}$ and $\mathrm{HW}$ wrote the manuscript.

\section{Acknowledgments}

We would like to thank the pathology support building staff for their support with animal care and Nigel Rust for assistance with cell sorting. This work was supported by the MRC UK (program grant G1000215) and the European Research Council (PARIS).

Address correspondence to: Duncan Howie, Sir William Dunn School of Pathology, University of Oxford, OX1 3RE, United Kingdom. Phone: 44.1865.275518; E-mail: duncan.howie@path.ox.ac.uk.

1. Fontenot JD, Rasmussen JP, Williams LM, Dooley JL, Farr AG, Rudensky AY. Regulatory T cell lineage specification by the forkhead transcription factor foxp3. Immunity. 2005;22(3):329-341.

2. Fontenot JD, Gavin MA, Rudensky AY. Foxp3 programs the development and function of CD4+CD25+ regulatory T cells. Nat Immunol. 2003;4(4):330-336.

3. Hori S, Nomura T, Sakaguchi S. Control of regulatory T cell development by the transcription factor Foxp3. Science. 2003;299(5609):1057-1061.

4. Kretschmer K, Apostolou I, Hawiger D, Khazaie K, Nussenzweig MC, von Boehmer H. Inducing and expanding regulatory T cell populations by foreign antigen. Nat Immunol. 2005;6(12):1219-1227.

5. Kendal AR, et al. Sustained suppression by Foxp3+ regulatory T cells is vital for infectious transplantation tolerance. J Exp Med. 2011;208(10):2043-2053.

6. Regateiro FS, et al. Foxp3 expression is required for the induction of therapeutic tissue tolerance. J Immunol. 2012;189(8):3947-3956.

7. Andersen KG, Butcher T, Betz AG. Specific immunosuppression with inducible Foxp3-transduced polyclonal T cells. PLoS Biol. 2008;6(11):e276.

8. Chen W, et al. Conversion of peripheral CD4+CD25- naive T cells to CD4+CD25+ regulatory T cells by TGF-beta induction of transcription factor Foxp3. J Exp Med. 2003;198(12):1875-1886.

9. Cobbold SP, et al. Induction of foxP3 + regulatory $\mathrm{T}$ cells in the periphery of $\mathrm{T}$ cell receptor transgenic mice tolerized to transplants. J Immunol. 2004;172(10):6003-6010.

10. Michalek RD, et al. Cutting edge: distinct glycolytic and lipid oxidative metabolic programs are essential for effector and regulatory CD4+ T cell subsets. J Immunol. 2011;186(6):3299-3303.

11. Daniel C, Weigmann B, Bronson R, von Boehmer H. Prevention of type 1 diabetes in mice by tolerogenic vaccination with a strong agonist insulin mimetope. J Exp Med. 2011;208(7):1501-1510.

12. Cobbold SP, et al. Infectious tolerance via the consumption of essential amino acids and mTOR signaling. Proc Natl Acad Sci USA. 2009;106(29):12055-12060. 
13. Berod L, et al. De novo fatty acid synthesis controls the fate between regulatory T and T helper 17 cells. Nat Med. 2014;20(11):1327-1333.

14. Gerriets VA, et al. Metabolic programming and PDHK1 control CD4+ T cell subsets and inflammation. J Clin Invest. 2015;125(1):194-207.

15. Cobbold SP, et al. Regulatory T cells and dendritic cells in transplantation tolerance: molecular markers and mechanisms. Immunol Rev. 2003;196:109-124.

16. Sadlon TJ, et al. Genome-wide identification of human FOXP3 target genes in natural regulatory T cells. J Immunol. 2010;185(2):1071-1081.

17. Marson A, et al. Foxp3 occupancy and regulation of key target genes during T-cell stimulation. Nature. 2007;445(7130):931-935.

18. Zheng Y, Josefowicz SZ, Kas A, Chu TT, Gavin MA, Rudensky AY. Genome-wide analysis of Foxp3 target genes in developing and mature regulatory T cells. Nature. 2007;445(7130):936-940.

19. Chang $\mathrm{CH}$, et al. Posttranscriptional control of T cell effector function by aerobic glycolysis. Cell. 2013;153(6):1239-1251.

20. van der Windt GJ, et al. Mitochondrial respiratory capacity is a critical regulator of CD8+ T cell memory development. Immunity. 2012;36(1):68-78

21. Xie X, et al. The Regulatory T Cell Lineage Factor Foxp3 Regulates Gene Expression through Several Distinct Mechanisms Mostly Independent of Direct DNA Binding. PLoS Genet. 2015;11(6):e1005251.

22. Chi H. Regulation and function of mTOR signalling in T cell fate decisions. Nat Rev Immunol. 2012;12(5):325-338.

23. Vogel C, Marcotte EM. Insights into the regulation of protein abundance from proteomic and transcriptomic analyses. Nat Rev Genet. 2012;13(4):227-232.

24. de Pablo MA, et al. Palmitate induces apoptosis via a direct effect on mitochondria. Apoptosis. 1999;4(2):81-87.

25. Wu Z, et al. Mechanisms controlling mitochondrial biogenesis and respiration through the thermogenic coactivator PGC-1. Cell. 1999;98(1):115-124

26. Mootha VK, et al. Erralpha and Gabpa/b specify PGC-1alpha-dependent oxidative phosphorylation gene expression that is altered in diabetic muscle. Proc Natl Acad Sci USA. 2004;101(17):6570-6575.

27. Beier UH, et al. Essential role of mitochondrial energy metabolism in Foxp3 ${ }^{+}$T-regulatory cell function and allograft survival. FASEB J. 2015;29(6):2315-2326.

28. Frauwirth KA, et al. The CD28 signaling pathway regulates glucose metabolism. Immunity. 2002;16(6):769-777.

29. Jacobs SR, et al. Glucose uptake is limiting in T cell activation and requires CD28-mediated Akt-dependent and independent pathways. J Immunol. 2008;180(7):4476-4486.

30. Roos D, Loos JA. Changes in the carbohydrate metabolism of mitogenically stimulated human peripheral lymphocytes. II. Relative importance of glycolysis and oxidative phosphorylation on phytohaemagglutinin stimulation. Exp Cell Res. 1973;77(1):127-135

31. Bental M, Deutsch C. Metabolic changes in activated T cells: an NMR study of human peripheral blood lymphocytes. Magn Reson Med. 1993;29(3):317-326.

32. Walker LS, Chodos A, Eggena M, Dooms H, Abbas AK. Antigen-dependent proliferation of CD4+ CD25+ regulatory T cells in vivo. J Exp Med. 2003;198(2):249-258

33. Pierson W, et al. Antiapoptotic Mcl-1 is critical for the survival and niche-filling capacity of Foxp3 $3^{+}$regulatory T cells. Nat Immunol. 2013;14(9):959-965

34. Basu S, Hubbard B, Shevach EM. Foxp3-mediated inhibition of Akt inhibits Glut1 (glucose transporter 1) expression in human T regulatory cells. J Leukoc Biol. 2015;97(2):279-283.

35. Macintyre AN, et al. The glucose transporter Glut1 is selectively essential for CD4 T cell activation and effector function. Cell Metab. 2014;20(1):61-72.

36. Procaccini C, et al. The Proteomic Landscape of Human Ex Vivo Regulatory and Conventional T Cells Reveals Specific Metabolic Requirements. Immunity. 2016;44(2):406-421

37. Lochner M, Berod L, Sparwasser T. Fatty acid metabolism in the regulation of T cell function. Trends Immunol. 2015;36(2):81-91.

38. Shrestha S, Yang K, Guy C, Vogel P, Neale G, Chi H. Treg cells require the phosphatase PTEN to restrain TH1 and TFH cell responses. Nat Immunol. 2015;16(2):178-187.

39. Huynh A, et al. Control of PI(3) kinase in Treg cells maintains homeostasis and lineage stability. Nat Immunol. 2015;16(2):188-196

40. Regateiro FS, et al. Generation of anti-inflammatory adenosine by leukocytes is regulated by TGF- $\beta$. Eur J Immunol. 2011;41(10):2955-2965.

41. Adra CN, et al. Cloning of the cDNA for a hematopoietic cell-specific protein related to CD20 and the beta subunit of the high-affinity IgE receptor: evidence for a family of proteins with four membrane-spanning regions. Proc Natl Acad Sci USA 1994;91(21):10178-10182.

42. Stagg J, et al. Anti-CD73 antibody therapy inhibits breast tumor growth and metastasis. Proc Natl Acad Sci USA 2010;107(4):1547-1552.

43. Jin D, et al. CD73 on tumor cells impairs antitumor T-cell responses: a novel mechanism of tumor-induced immune suppression. Cancer Res. 2010;70(6):2245-2255.

44. Wang L, et al. CD73 has distinct roles in nonhematopoietic and hematopoietic cells to promote tumor growth in mice. J Clin Invest. 2011;121(6):2371-2382.

45. Bastid J, Cottalorda-Regairaz A, Alberici G, Bonnefoy N, Eliaou JF, Bensussan A. ENTPD1/CD39 is a promising therapeutic target in oncology. Oncogene. 2013;32(14):1743-1751.

46. Reaven GM, Hollenbeck C, Jeng CY, Wu MS, Chen YD. Measurement of plasma glucose, free fatty acid, lactate, and insulin for $24 \mathrm{~h}$ in patients with NIDDM. Diabetes. 1988;37(8):1020-1024.

47. Risé P, Eligini S, Ghezzi S, Colli S, Galli C. Fatty acid composition of plasma, blood cells and whole blood: relevance for the assessment of the fatty acid status in humans. Prostaglandins Leukot Essent Fatty Acids. 2007;76(6):363-369.

48. Richieri GV, Kleinfeld AM. Unbound free fatty acid levels in human serum. J Lipid Res. 1995;36(2):229-240.

49. Ulloth JE, Casiano CA, De Leon M. Palmitic and stearic fatty acids induce caspase-dependent and -independent cell death in nerve growth factor differentiated PC12 cells. J Neurochem. 2003;84(4):655-668. 
50. Sharma RB, Alonso LC. Lipotoxicity in the pancreatic beta cell: not just survival and function, but proliferation as well? Curr Diab Rep. 2014;14(6):492.

51. Eller K, et al. Potential role of regulatory T cells in reversing obesity-linked insulin resistance and diabetic nephropathy. Diabetes 2011;60(11):2954-2962.

52. Zeyda M, Huber J, Prager G, Stulnig TM. Inflammation correlates with markers of T-cell subsets including regulatory T cells in adipose tissue from obese patients. Obesity (Silver Spring). 2011;19(4):743-748.

53. Pereira S, et al. Modulation of adipose tissue inflammation by FOXP3 + Treg cells, IL-10, and TGF- $\beta$ in metabolically healthy class III obese individuals. Nutrition. 2014;30(7-8):784-790.

54. Feuerer M, et al. Lean, but not obese, fat is enriched for a unique population of regulatory T cells that affect metabolic parameters. Nat Med. 2009;15(8):930-939.

55. Deiuliis J, et al. Visceral adipose inflammation in obesity is associated with critical alterations in tregulatory cell numbers. $P L o S$ One. 2011;6(1):e16376

56. Ma C, et al. NAFLD causes selective CD4(+) T lymphocyte loss and promotes hepatocarcinogenesis. Nature. 2016;531(7593):253-257.

57. Xu S, et al. Palmitate induces ER calcium depletion and apoptosis in mouse podocytes subsequent to mitochondrial oxidative stress. Cell Death Dis. 2015;6:e1976.

58. Guo W, Jiang L, Bhasin S, Khan SM, Swerdlow RH. DNA extraction procedures meaningfully influence qPCR-based mtDNA copy number determination. Mitochondrion. 2009;9(4):261-265.

59. Boersema PJ, Raijmakers R, Lemeer S, Mohammed S, Heck AJ. Multiplex peptide stable isotope dimethyl labeling for quantitative proteomics. Nat Protoc. 2009;4(4):484-494.

60. Trudgian DC, et al. Comparative evaluation of label-free SINQ normalized spectral index quantitation in the central proteomics facilities pipeline. Proteomics. 2011;11(14):2790-2797.

61. Lui KO, Howie D, Ng SW, Liu S, Chien KR, Waldmann H. Tolerance induction to human stem cell transplants with extension to their differentiated progeny. Nat Commun. 2014;5:5629.

62. Vizcaíno JA, et al. ProteomeXchange provides globally coordinated proteomics data submission and dissemination. Nat Biotech nol. 2014;32(3):223-226

63. Salabei JK, Gibb AA, Hill BG. Comprehensive measurement of respiratory activity in permeabilized cells using extracellular flux analysis. Nat Protoc. 2014;9(2):421-438.

64. Campos CB, Paim BA, Cosso RG, Castilho RF, Rottenberg H, Vercesi AE. Method for monitoring of mitochondrial cytochrome $\mathrm{c}$ release during cell death: Immunodetection of cytochrome $\mathrm{c}$ by flow cytometry after selective permeabilization of the plasma membrane. Cytometry A. 2006;69(6):515-523. 